\title{
A method of multiscale modelling considered as a way leading to unified mechanics of materials
}

Received: 9 June 2014 / Revised: 2 September 2014 / Published online: 25 November 2014

(C) The Author(s) 2014. This article is published with open access at Springerlink.com

\begin{abstract}
This paper outlines a development of rational mechanics which may potentially lead to a more unified mechanics of materials. The intention of considerations is to provide in a future a theoretical context for systematic engineering activity in various scales. Methodological considerations leading to a development on the above way are the first aim of this paper. We consider the multiscale method of modelling called collection of dynamical systems with dimensional reduction. Within this approach, the scale of averaging applied in modelling is formalized. Therefore transition between various scale models is possible. In order to make the dimensional reduction procedure realizable, we have to formulate continuum mechanics with finitedimensional fields in connection with an elementary dynamical system. Such a step is also aimed at obtaining the possibility of the consideration of atomistic and continuum models within one consistent theoretical scheme. One accentuates the particular role of nanoscale models as a bridge between atomistic models and more averaged continuum description. One discusses the methodology of nanoscale modelling accentuating the particular role of the free energy and methodology of defining this function.
\end{abstract}

\section{Introduction}

Contemporary engineering is developed in many directions and in relation to various scales. Efficient design of constructions and manufacturing processes depends on theoretical models which we apply to this end. In particular, the state of theory determines also the possibility of automatizing processes of design by means of computers.

We observe such a situation in case of engineering related to scale corresponding to traditional constructions in our environment where the theory applied is predominantly well developed. However, we observe also a tendency in engineering towards decreasing of scale where design of devices is carried out. The most outstanding example of this tendency is the promotion of nanotechnology. Frequently, design of nanotechnological devices has to be based on experience of scientists realizing experiments in a corresponding field since theory is not so effective in small scale as this is the case for traditional constructions.

Let us notice that we can support efficiently the design process by numerical simulations when construction works in elastic range. In case of transition to inelastic deformation for instance, we can also apply many models. However, then the question on the range of validity of the equations appears. Frequently, support from experiment is then necessary. In such a case, theory and numerical simulations have rather auxiliary character. This means that we have not sufficient confidence in models of mechanics of materials when more complex phenomena based on less clear physical processes appear. Such difficulties will increase when we would like to design devices and material functions in relation to nanoscale.

J. Kaczmarek $(\varangle)$

Institute of Fluid-Flow Machinery, Polish Academy of Sciences, ul. J.Fiszera 14, 80-231 Gdańsk, Poland

E-mail: jarekk@imp.gda.pl 
Summarizing, we would like to have at our disposal the possibility of design in all necessary scales. An obstacle on this way is the necessity of determination of the range of validity of the theoretical model applied. Usually, equations, especially related to mechanics of materials, have not precisely defined their range of validity. This in turn suggests that the method of derivation of such equations is related to not entirely clear methodology and perhaps physical foundations.

In order to overcome such a difficulty, we should have at our disposal a kind of unified mechanics of materials in relation to various scales. Furthermore, the scale of averaging of properties of corresponding processes within the model should be clearly defined. Within unified mechanics containing various cooperating models, the range of validity of each model should be determined. This in turn is a necessary condition for carrying out consistent numerical simulations of the design process. Transition between various models during computation could be realized by means of critical conditions for the validity of particular models automatically. Then, numerical simulations could be realized efficiently in the whole range of activity of physical processes in which the designed process is involved.

The above discussion suggests the necessity of having at our disposal a kind of unified mechanics of materials based on clear mathematical foundations and valid for a large range of phenomena. Let us note that in the past we had to do with various attempts in theoretical mechanics which have similar aims. On the one hand, the rational mechanics is constructed in order to provide theory based on a clear system of mathematical assumptions in relation to continuum description [1]. On the other hand, statistical mechanics has tried to provide more physical foundations, also for continuum mechanics, basing on atomistic calculations [2,3].

The rational mechanics is a successful step in mathematical formalization of continuum mechanics. However, its further development consists, to a higher degree, in transformation to a kind of mathematical mechanics. It rests on application of various methods of mathematics in mechanics instead of constructing a consistent unified theory. An obstacle on this way is related especially to the theory of constitutive equations. We observe a division of mechanics of materials into parts related to particular phenomena, and the tendency to unification is not too strong.

On the other hand, the statistical mechanics creates physical foundations for various theoretical models. However, wide application of statistical mechanics encounters some obstacles related to complexity of atomistic simulations. In particular, it is difficult to create models with larger scale of averaging by means of statistical mechanics where many intermediate scale processes take place. The complexity of such processes makes difficult the elaboration of appropriate methods of averaging.

We would say that there is a gap between statistical mechanics and rational mechanics. This gap is related to the difficulty with averaging over many physical processes responsible for instance for inelastic deformation which take place between atomistic scale and scale related to a traditional continuum. Let us also note that the scale of averaging related to continuum models is usually not precisely specified.

The last aspect follows from the fact that within the rational mechanics the scale of averaging is not formalized. Consequently, going towards unified theoretical mechanics, we should formalize the scale of averaging.

Scales of averaging applied in continuum models are usually larger than microscale. Let us consider the crystal plasticity as example where the scale of averaging is taken into account. Within this approach, the scale is not formalized. This scale is manifested by objects which are modelled. Let us mention slip bands for instance in case of crystal plasticity. Averaging over slip surfaces suggests that corresponding models are related to microscale. However, many mechanisms of inelastic deformation are related to nanoscale. They are associated for instance with single slip surface or single martensite variants and corresponding separate interfaces. It suggests in turn that nanoscale models could be important.

Let us note that nanoscale models, important with respect to the discussed above mechanisms of inelastic deformation, are not constructed in fact. Some nanoscale models for martensitic transformation for instance exist without formalization of scale. Some others related to dislocation theory could be considered also as nanoscale. However, a formal approach to modelling within this scale is not continued for several reasons. First, the scale of averaging is not formalized. Secondly, corresponding nanoscale models are not well justified with respect to applications. In engineering practice, we have to do rather with simpler and more averaged models. Nanoscale models should be complicated. Then, we should justify what for such models should be produced.

It seems that nanoscale models should fill the gap between atomistic models and the continuum models related to larger scale. We should elaborate a method of transition between various scale models. Then, transition from atomistic model to nanoscale model should be relatively simpler than directly to larger-scale model since nanoscale models are connected with smaller representative volume. Transition to larger-scale 
model would be continued from nanoscale models. Thereby, nanoscale models could obtain its own status. Furthermore, the increasing role of nanotechnology provides a new area of applications directly for nanoscale models. The above discussion promotes in fact a multiscale description within mechanics of materials with particular status of nanoscale models.

Let us also note that within mechanics of materials micromechanics is developed [4,5]. This is a kind of multiscale mechanics. By means of micromechanics, we derive more averaged models of mechanics of materials from premises related to a smaller scale.

Internal state variables are also applied for an averaged description of effects in materials following from smaller scale. Efforts for formalizing such an approach within mechanics of materials and in context of the rational mechanics are described in [6].

Let us note that with application of decreasing scale averaging during modelling, the role of dynamics of processes increases. Thereby, smaller scale models should have larger connections with dynamical systems. This suggests that we should promote a multiscale description which is based on the dynamical system approach.

In this paper, one proposes various methods of modelling having the intention of obtaining in a future a kind of unified theory of mechanics of materials. The presented approach is based on multiscale modelling called here collection of dynamical systems with dimensional reduction. Formalization of scale of averaging is introduced. The role of nanoscale models is presented by discussion of an example of nanoscale model of martensitic transformation conjugated with nanoscale model of slip plasticity. One accentuates methodological aspects of modelling especially related to methods of defining the free energy.

The method of theoretical description discussed in this paper is considered as a way of development of rational mechanics towards incorporation of discrete models related to atomic scale and averaged continuum models into one theoretical framework.

Within this paper, I try to promote the point of view that unified mechanics of materials should be constructed taking into account two aspects. The first one states that the developed theory should be a well-defined mathematical theory within which precise and formal considerations could be carried out. The second aspect has to some degree opposite character. It states that the material modelled is physical structure present within real world. Then, the theory applied approximates its behaviour only. Therefore, it is important to discuss, within the applied model, the methodology of approximation of reality and by this status of applied mathematical methods. There is a hope that within further development of unified mechanics of materials the methods of approximation of reality will be systematized and also expressed in formal way.

Let us note that rational mechanics as such evolves rather towards mathematical mechanics neglecting to large degree the methodology of approximation of reality.

The main aim of this paper is to elucidate a methodology which could be applied on the way leading towards unified mechanics of materials within one work which is based on earlier papers of the author. One accentuates the status of various theories within this approach. It is also suggested how continuum mechanics could become a more physical theory by joining it by multiscale approach with more fundamental physical theories. By this step, we promote in fact the interdisciplinary character of mechanics of materials.

\section{Multiscale modelling by collection of dynamical systems with dimensional reduction}

\subsection{Introduction of collection of dynamical systems}

With decreasing scale, we observe an increasing role of dynamics of processes. This suggests using a dynamical system approach when we would like to describe the most elementary processes in small scale. Dynamical systems theory is an important branch of mathematics [7] directed towards modelling of the evolution of systems and is widely applied in physics and mechanics, see for instance [8]. Our considerations in this section are based on $[9,10]$.

We expect considerable complexity of phenomena related to small scale. Not all properties of these processes are interesting for us. Thereby, we would like to have at our disposal some methods of simplification of this complex physical picture in order to refine properties more interesting for us of the system modelled. A possible way for such a simplification is to distinguish some dynamical subsystems. Then, the obtained structure of dynamical subsystems would be a base for the dimensional reduction procedure. 
We introduce first a dynamical system aimed at the description of phenomena on the most elementary level. Such a system is called here the elementary dynamical system (EDS) and is given in a general form

$$
\dot{\varphi}=L(\boldsymbol{\varphi}, \mathbf{f})
$$

where $\varphi \in \mathcal{M}_{\varphi}$ is the variable of this system, $\mathcal{M}_{\varphi}$ is a space of admissible values of this variable, $\mathbf{f} \in \mathcal{F}$ represents external interactions acting on this system, and $\mathcal{F}$ stands for the space of admissible values of $\mathbf{f}$.

External interactions are not always expressed in a simple form given by $\mathbf{f}$. Sometimes, they appear as interactions with other dynamical systems. Therefore, we introduce also an extended dynamical system

$$
\dot{\varphi}^{r}=L\left(\varphi^{r}, \mathbf{f}^{r}\right)
$$

where $\varphi^{r}=\left\{\boldsymbol{\varphi}, \varphi^{e}\right\} \in \mathcal{M}_{\varphi r}$ and $\mathbf{f}^{r}=\left\{\mathbf{f}, \mathbf{f}^{e}\right\} \in \mathcal{F}_{r}$. In other words, the dynamical system (1) is a part of that one defined by (2) and can be viewed as a particular case of (2). As a result of this an external dynamical system with variable $\varphi^{e}$ can be additionally distinguished, as a model of external interactions acting on EDS. This gives a possibility of discussing a larger class of interactions of (1) with an external world.

The dynamical system (1) describes more elementary processes and its form is, by assumption, the most complex. We tend towards simplifications of this system. To this end, we introduce a partition of (1) onto a collection of $P$ dynamical systems. This is carried out by partition of variable $\varphi=\left\{\varphi_{h}\right\}, h \in I_{P}=$ $\{1,2, \ldots, P\}$.

In order to use this partition for further simplifications, we introduce also additional notations, sets and mappings. Let $\prod_{h} \mathcal{M}_{h}$ be the Cartesian product of sets $\mathcal{M}_{h}$, where $\mathcal{M}_{h}$ stands for the set of admissible values of $\boldsymbol{\varphi}_{h}$. Let us introduce $\mathcal{M}_{\Pi} \subset \prod_{h} \mathcal{M}_{h}$ as a subset of the Cartesian product. Then, $\mathcal{M}_{\Pi}$ consists of $\boldsymbol{\varphi}=\left\{\boldsymbol{\varphi}_{h}\right\}$ which are possible solutions of Eq. (1). We consider also a projection $\pi_{h}: \mathcal{M}_{\Pi} \rightarrow \mathcal{M}_{h}$ in the Cartesian product.

Let us distinguish also a collection of dynamical systems for the extended system and a set of indexes $I_{R}$ related to them. Then, $I_{R}=I_{P} \cup I_{E}, I_{P} \cap I_{E}=\emptyset$, where $I_{P}$ is related to (1) and $I_{E}=I_{R}-I_{P}$ is connected with the external system. Furthermore, we distinguish a group of dynamical subsystems $I_{G} \subset I_{P}$ by selection of corresponding indexes. Then, $I_{O}=I_{R}-I_{G}$ represents indexes defining external with respect to $I_{G}$ dynamical subsystems within (2).

New simplified equations have to be based on balance of mass and energy equations as the most fundamental physical laws for the mechanics of materials. In order to create such equations for the collection of dynamical systems, we should have at our disposal a set of additional notions making possible to formulate them. Therefore, we introduce the following assumptions which represent general properties of the collection of dynamical systems distinguished within the elementary dynamical system [9]:

1. Subsystems are distinguished by the determination of groups of variables $\varphi_{h}=\left\{\varphi_{h \alpha}\right\}$, where $\alpha \in I_{\mathrm{Ah}}, I_{\mathrm{Ah}}$ is a finite set related to the separate $h$ th subsystem and $h \in I_{P}$.

2. There exists a function $\bar{m}_{h}\left(\boldsymbol{\varphi}_{h}\right)=\left\{m_{h 1}, \ldots, m_{h \beta_{h}}\right\}$ which assigns a set of masses for the $h$ th subsystem. The total mass of the system is $m_{h}=\sum_{i} m_{h i}$. We have also that $\sum_{h} \beta_{h}=N$, where $N$ is the total number of masses. Then, the functions $\tilde{m}: \mathcal{M}_{\Pi} \rightarrow R^{P}$ with property $\pi_{h} \circ \tilde{m}\left(\left\{\boldsymbol{\varphi}_{h}\right\}\right)=m_{h}$ and $m: \mathcal{M}_{\Pi} \rightarrow$ $R, m\left(\left\{\boldsymbol{\varphi}_{h}\right\}\right)=\sum m_{h}$ determine the total mass related to each subsystem and the total mass related to (1), respectively.

3. There exists a function $\tilde{E}: \mathcal{M}_{\Pi} \rightarrow R^{P}, \pi_{h} \circ \tilde{E}\left(\left\{\boldsymbol{\varphi}_{h}\right\}\right)=E_{h}$ which assigns a value of energy to each of the subsystems and $E: \mathcal{M}_{\Pi} \rightarrow R, E\left(\left\{\boldsymbol{\varphi}_{h}\right\}\right)=\sum_{h} E_{h}$ determines the total energy related to (1).

4. There exists a family of mappings $J_{i j}: \mathcal{M}_{\Pi} \rightarrow R, i, j \in I_{P}, J_{i j}\left(\left\{\varphi_{h}\right\}\right)=J_{i j}$ called flux of mass from $j$ th subsystem to $i$ th subsystem and $J_{i j}+J_{j i}=0, J_{i i}=0$.

5. There exists a family of mappings $W_{i j}: \mathcal{M}_{\Pi} \rightarrow R, i, j \in I_{P}, W_{i j}\left(\left\{\boldsymbol{\varphi}_{h}\right\}\right)=W_{i j}$ called flux of energy from $j$ th subsystem to $i$ th subsystem and $W_{i j}+W_{j i}=0, W_{i i}=0$.

6. A source of mass is determined by the function $c: \mathcal{M}_{\Pi} \rightarrow R^{P}, c\left(\left\{\boldsymbol{\varphi}_{h}\right\}\right)=\left\{c_{i}\right\}$. Then, $c_{i}=\pi_{i} \circ c\left(\left\{\boldsymbol{\varphi}_{h}\right\}\right)$ can be considered for each subsystem of the whole system and stands for the source of mass in $i$ th subsystem.

7. A source of energy is determined by the function $R: \mathcal{M}_{\Pi} \rightarrow R^{P}, R\left(\left\{\varphi_{h}\right\}\right)=\left\{R_{i}\right\}$. Then, $R_{i}=$ $\pi_{i} \circ R\left(\left\{\varphi_{h}\right\}\right)$ can be considered for each subsystem of the whole system and stands for the source of energy in $i$ th subsystem.

8. Geometrical objects can be assigned to each subsystem. This is carried out with the help of mappings $G_{x}: \mathcal{M}_{\Pi} \rightarrow E_{e}^{P}, G_{L}: \mathcal{M}_{\Pi} \rightarrow\left(2^{E_{e}}\right)^{P}, G_{S}: \mathcal{M}_{\Pi} \rightarrow\left(2^{E_{e}}\right)^{P}, G_{V}: \mathcal{M}_{\Pi} \rightarrow\left(2^{E_{e}}\right)^{P}$, where $E_{e}$ is the Euclidean space. The map $G_{x}$ assigns some distinguished points to subsystems, $G_{L}$ introduces 
one-dimensional, $G_{S}$ two-dimensional, $G_{V}$ three-dimensional geometrical objects considered as subsets of $E_{e}$ and accompanied by distinguished subsystems.

All discussed assumptions and functions can also be introduced for the extended dynamical system (2). They enable us considerations of balance of mass and energy equations for the collection of dynamical systems. Geometrical elements allow us to create various relations associated with geometry of our space in relation to dynamical subsystems.

\subsection{Balance of mass and energy for collection of dynamical systems}

By means of the assumptions introduced above, we are able to carry out an analysis of interchange of mass between subsystems as well as to consider possible sources of mass which appear within subsystems. Then, we are able also to express the balance of mass equation for collection of dynamical systems in the following form:

$$
\sum_{i \in I_{G}}\left(\dot{m}_{i}-c_{i}\right)+\sum_{i, j \in I_{G}} J_{i j}+\sum_{i \in I_{G}, j \in I_{O}}\left(J_{i j}+J_{j i}\right)+\sum_{i, j \in I_{O}} J_{i j}+\sum_{i \in I_{O}}\left(\dot{m}_{i}-c_{i}\right)=0 .
$$

The sum of masses interchanged between subsystems within $I_{G}$ without any interchange with an external subsystems is equal to zero. Thus, we have $\sum_{i, j \in I_{G}} J_{i j}=0$. As a result, we can express the balance of mass equation connected with the group of subsystems represented by $I_{G}$ with the help of formula

$$
\sum_{i \in I_{G}}\left(\dot{m}_{i}-c_{i}+\sum_{j \in I_{O}} J_{i j}\right)=0
$$

In Eq. (4), the terms $J_{i j}$ describing the interchange of mass with external system $I_{O}$ appear. Then, Eq. (4) is not entirely determined. Therefore, we should introduce an additional condition

$$
J_{i j}=\bar{J}_{i j}, \quad j \in I_{O}
$$

The form of $\bar{J}_{i j}$ should be postulated by a kind of constitutive equations.

The balance of energy equation has similar structure as the balance of mass equation and is given by

$$
\sum_{i \in I_{G}}\left(\dot{E}_{i}-R_{i}\right)+\sum_{i \in I_{G}, j \in I_{O}}\left(W_{i j}+W_{j i}\right)+\sum_{i \in I_{O}}\left(\dot{E}_{i}-R_{i}\right)=0 .
$$

The balance of energy equation for the group of subsystems $I_{G}$ interacting with the groups of subsystems $I_{O}$, is given by

$$
\sum_{i \in I_{G}}\left(\dot{E}_{i}-R_{i}+\sum_{j \in I_{O}} W_{i j}\right)=0
$$

with additional conditions

$$
W_{i j}=\bar{W}_{i j}, \quad j \in I_{O}
$$

Let us note that $E_{i}$ and $W_{i j}$ depend, in general, on the state of the whole system in accordance with Assumptions 3 and 5.

Equations (4), (5) and (7), (8) represent a general form of balance of mass and energy equations related to an arbitrary distinguished group of subsystems within the collection of dynamical systems.

We consider here balance of mass and energy equations only. The balance of momentum equation is seen here as law which is a consequence of the balance of mass and energy equations which sometimes can be postulated independently. 


\subsection{Description of the dimensional reduction procedure}

The dimensional reduction procedure is a method of transition from an elementary dynamical system to a simpler dynamical system having a smaller number of degrees of freedom.

The first step of the dimensional reduction consists in the option of new variables. Let $\mathbf{d}=\left\{\mathbf{d}_{h}\right\}, h \in I_{R}$ be a set of new variables which allows to describe, approximately, physical states represented by miscellaneous $\varphi^{r}$. Let $\overline{\mathcal{M}}$ stand for the space of admissible values of $\mathbf{d}$. $\mathbf{d}_{h}$ describes the behaviour of $h$ th subsystem in a simplified form. We have by assumption that $\operatorname{dim} \overline{\mathcal{M}}$ is considerably smaller than $\operatorname{dim} \mathcal{M}_{\varphi}$.

Let $V_{T}=\{\varphi(t): t \in T\}, V_{T r}=\left\{\varphi^{r}(t): t \in T\right\}$ and $\bar{V}_{T}=\{\mathbf{d}(t): t \in T\}$. The first element of the dimensional reduction procedure is based on the introduction of a mapping $\pi_{T}: V_{T r} \rightarrow \bar{V}_{T}$ which assigns the dimensionally reduced process $\mathbf{d}(t)$ to $\varphi^{r}(t)$ on the time interval $T$.

We introduce also $\mathcal{F}_{T}=\{\mathbf{f}(t), t \in T\}$ and $\overline{\mathcal{F}}_{T}=\{\overline{\mathbf{f}}(t), t \in T\}$ with mapping $\pi_{f T}: \mathcal{F}_{T} \rightarrow \overline{\mathcal{F}}_{T}$ which transforms forces between elementary and reduced system.

We can introduce a division of variables $\mathbf{d}(t)$ into parts $\mathbf{d}^{p}(t)$ and $\mathbf{d}^{e}(t)$ as corresponding with EDS and external dynamical system in smaller scale. A similar partition is possible for $\mathbf{f}(t)$ and $\mathbf{f}(t)$ since they are considered here for the extended system. Thus, we carry out the dimensional reduction for the extended dynamical system (2). However, our interest is related mainly to EDS given by (1).

The external dynamical system is purposed to model a larger class of external interactions. Thereby, the external interactions related to EDS are given by $\mathbf{f}(t)$ and $\overline{\mathbf{f}}(t)$ and furthermore by the form of the external system. In particular, such a system can be reduced to the most simple form represented by static forces. Thus, the dimensional reduction procedure should represent different procedures for EDS and for the external system in general. This takes place since the discussed systems have separate status in the framework of the presented description. However, we will use $\varphi$ instead of $\varphi^{r}$ and $\mathbf{f}$ instead of $\mathbf{f}^{r}$ in what follows if it does not lead to mistake.

Let us introduce an operator $\mathcal{L}: \mathcal{M}_{\varphi r} \rightarrow \mathcal{F}_{r}$ constructed with the help of Eq. (2) as $\mathcal{L}(\varphi)=\tilde{\mathcal{L}}(\varphi, \dot{\varphi})$, where $\tilde{\mathcal{L}}$ is obtained from an equivalent to (2) equation in the form $\tilde{\mathcal{L}}(\varphi, \dot{\varphi})=\mathbf{f}^{r}$. Then, the operator acting on processes $\mathcal{L}_{T}: V_{T r} \rightarrow \overline{\mathcal{F}}_{T}$ is induced directly by means of $\mathcal{L}$ for each $t \in T$. Let us consider a diagram

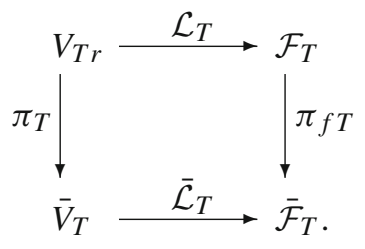

Accordingly, the initially introduced equation $\mathcal{L}_{T}(\varphi(t))=\mathbf{f}(t)$ induces, owing to the assumed $\pi_{T}$ and $\pi_{f T}$, a dimensionally reduced equation

$$
\overline{\mathcal{L}}_{T}(\mathbf{d}(t))=\overline{\mathbf{f}}
$$

where $\overline{\mathcal{L}}_{T}=\pi_{f T} \circ \mathcal{L}_{T} \circ \pi_{T}^{-1}$. The operator $\overline{\mathcal{L}}_{T}$ can be determined with the help of solutions of Eq. (2) and postulated mappings $\pi_{T}, \pi_{f T}$ for each value of $\mathbf{d}(t)$. However, the structure of the operator $\overline{\mathcal{L}}_{T}$ is not discussed yet since a too small number of assumptions is done at this moment.

Let us discuss a similar diagram for a group of subsystems $I_{G} \subset I_{P}$. Then, we carry out the following decomposition $V_{T r}=V_{T G} \times V_{T O}=\left\{\boldsymbol{\varphi}(t)=\left\{\boldsymbol{\varphi}_{g}(t), \boldsymbol{\varphi}_{l}(t)\right\}, g \in I_{G}, l \in I_{O}\right\}$. The symbol " $\times$ " does not stand for Cartesian product operation but means a kind of relation in which $\varphi_{g}, \varphi_{l}$ together create a solution of (2). We introduce also decomposition $\mathcal{F}_{T}=\mathcal{F}_{T G} \times \mathcal{F}_{T O}$ in a similar way.

Owing to the above decompositions of domain and range, the operator $\mathcal{L}_{T}$ can be expressed as $\mathcal{L}_{T}=$ $\mathcal{L}_{T\left(\varphi_{l}\right)}\left(\left\{\boldsymbol{\varphi}_{g}(t)\right\}\right) \times \mathcal{L}_{T\left(\varphi_{g}\right)}\left(\left\{\boldsymbol{\varphi}_{l}(t)\right\}\right)$.

The operator $\mathcal{L}_{T\left(\varphi_{l}\right)}: V_{T G} \rightarrow \mathcal{F}_{T G}$ depends on $\varphi_{l}$. Usually, not all $\varphi_{l} \in V_{T O}$ are necessary for determination of $\mathcal{L}_{T\left(\varphi_{l}\right)}$.

Let $I_{G O}$ stand for a set of indexes which indicate variables necessary for the determination of the operator $\mathcal{L}_{T G}:=\mathcal{L}_{T\left(\varphi_{l}\right)}$. Then, the operator $\mathcal{L}_{T}$ can be expressed in simplified form as $\mathcal{L}_{T G} \times T_{C}$, where $T_{C}=$ $T_{C}\left(\varphi_{g}, \varphi_{c}\right), c \in I_{G O}$. The operator $T_{C}$ can have considerably simpler form for the determination of external 
interactions in comparison with $\mathcal{L}_{T}$. With the help of $T_{C}$, we are able to introduce additional equations necessary for the determination of $\boldsymbol{\varphi}_{c}$ defining form of $\mathcal{L}_{T G}$ within $\mathcal{L}_{T}$. The space $V_{T r}$ is reduced to $V_{T G} \times V_{T C}$ in this case.

The modified diagram (9) can be expressed now in the following form:

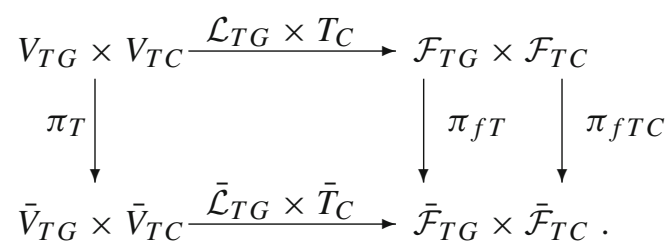

Accordingly, the dimensionally reduced equation describing the evolution of group of subsystems is given by

$$
\begin{aligned}
\overline{\mathcal{L}}_{T G\left(\mathbf{d}_{c}\right)}\left(\left\{\mathbf{d}_{g}(t)\right\}\right) & =\left\{\overline{\mathbf{f}}_{g}\right\}, \\
\bar{T}_{C c}\left(\mathbf{d}_{g}(t), \mathbf{d}_{c}(t)\right) & =\mathcal{B}_{c} .
\end{aligned}
$$

Diagram (11) allows to solve Eqs. (12), (13) by means of solutions of Eq. (2) and postulated form of $\pi_{T}, \pi_{f T}, \pi_{f T c}$. However, we would like to have a precise structure of $\overline{\mathcal{L}}_{T G}$ and $\bar{T}_{C}$ in order to have at our disposal possible to solution dimensionally reduced equations without using (2) incessantly.

We suggest to obtain corresponding equations by means of postulating the skeletal dynamical system SDS $(\mathbf{C})$ which depends on the family of constants $\mathbf{C}$. The role of SDS is to represent a larger class of systems which encompass approximately Eqs. (12) and (13). Accordingly, the general form of SDS in case of (12) and (13) can be expressed as $\left\{\overline{\mathcal{L}}_{T G}, \bar{T}_{C}\right\}(\mathbf{C})\left(\mathbf{d}_{g}, \mathbf{d}_{c}\right)=\{\overline{\mathbf{f}}, \mathcal{B}\}$. Then, with the help of an identification method, we can determine $\mathbf{C}=\overline{\mathbf{C}}$, and as a result of this, we obtain also a reduced dynamical system $\operatorname{RDS}=\operatorname{SDS}(\overline{\mathbf{C}})$. Finally, RDS represents an approximation of Eqs. (12) and (13).

The general structure of SDS should take into account fundamental laws in the first stage of formulation. In case of mechanics of materials, such laws are expressed by balance of energy and balance of mass equations. They have been previously formulated for a collection of dynamical systems.

A kind of variables $\mathbf{d}=\left\{\mathbf{d}_{h}\right\}$ also decides on the form of SDS. Furthermore, interactions between subsystems should be expressed by variables. To this end, we introduce a function of kinematical dependence between subsystems.

Let $H_{\mathrm{dh}}=\left\{\mathbf{d}_{j}: j \in I_{h}^{a}\right\}$ be a set of values of the variable $\mathbf{d}_{j}$ determined on a set of subsystems indexed by elements of a set $I_{h}^{a} . I_{h}^{a}$ represents all subsystems which interact with the $h$ th one.

Let us introduce a function $a_{h}: H_{d h} \rightarrow V_{a h}$, where $V_{a h}$ is a linear space. Accordingly, the function $a_{h}$ assigns an element of the linear space connected with $h$ th subsystem a set of values of variables $\mathbf{d}_{j}$ related to interacting subsystems. The function $a_{h}$ is called the function of kinematical dependence between subsystems. The form of this dependence is embodied in elements of $V_{a h}$.

We introduce also an additional concept of taking into account interactions between subsystems. Let us consider a discrete set of variables $\left\{g_{h}\right\}$ related to a dimensionally reduced system. In order to describe interactions between different systems, a value of $g_{h}$ type in a given point $\mathbf{X}$ can be useful. Consequently, we admit the possibility of introducing this kind of quantity. We assume that it is possible to introduce a transmission function $\mathcal{T}_{x}\left(\left\{g_{h}\right\}\right)=g_{x}$ which assigns a value of $g_{x}$ in the point $\mathbf{X}$ to the set of values $\left\{g_{h}\right\}$. We introduce $g_{x}$ as dependent on $\left\{g_{h}\right\}$. Consequently, no additional degrees of freedom are produced by this kind of function.

The transmission function can be introduced also more generally without interpretation of variables in the context of space position. Then, $\mathcal{T}_{\alpha}\left(\left\{g_{h}\right\}\right)=g_{\alpha}$ assigns additional variables to the set of existing variables. Sometimes such transformation allows to model interactions in a more convenient way.

Application of this function is possible with the help of the function $a_{h}$ which depends on the set of values of fields distributed in space and describe just interactions. In particular, the function $a_{h}$ can also be defined with the help of $g_{x}$ or $g_{\alpha}$.

Masses related to a dynamical system also undergo dimensional reduction. This is introduced by $\pi_{M}\left(\left\{m_{h i}\right\}\right)$ $=\left\{M_{h p}\right\}$. Thus, $M_{h p}$ are inertia coefficients related to the $h$ th subsystem and are present in the form of SDS.

Summing up these considerations, we have obtained some general premises for formulating the skeletal dynamical system. First, the balance of energy and mass equations should be taken into account. Next, quantities 
which appear in these equations should be plotted with $\mathbf{d}_{h}$ and $a_{h}(\mathbf{d})$ as well as with new inertia characteristics, in order to express state and interactions of subsystems. The transmission functions can be helpful to this end.

Let us discuss a concept of an identification method of constants defining RDS from $\operatorname{SDS}(\mathbf{C})$. Let $C=$ $\{\psi(t): \psi \in \overline{\mathcal{M}}, t \in T\}$ be a space of continuous time processes in $\overline{\mathcal{M}}$ with a metric $\rho: C \times C \rightarrow R_{+} \cup\{0\}$. We can construct two kinds of processes. The first one is based on solution $\varphi\left(\varphi_{0}, \mathbf{f}\right)(t)$ of Eq. (2) and has the form $\pi_{T}\left(\varphi\left(\varphi_{0}, \mathbf{f}\right)(t)\right)$. The second one is created by the skeletal dynamical system with assumed constants $\mathbf{C}$. Thus, we have a solution of equations of SDS as $\mathbf{d}\left(\mathbf{C}, \pi\left(\varphi_{0}\right), \overline{\mathbf{f}}\right)(t)$, where $\overline{\mathbf{f}}(t)=\pi_{f T}(\mathbf{f}(t))$. Let us consider the function

$$
h\left(\boldsymbol{\varphi}_{0}, \mathbf{f}\right)=\inf _{\mathbf{C} \in \mathcal{C}_{E}} \rho\left(\mathbf{d}\left(\mathbf{C}, \pi\left(\boldsymbol{\varphi}_{0}\right), \overline{\mathbf{f}}\right)(t), \pi_{T}\left(\boldsymbol{\varphi}\left(\boldsymbol{\varphi}_{0}, \mathbf{f}\right)(t)\right)\right)
$$

where $\mathcal{C}_{E}$ is an admissible set of constants for which the energy of the system is well approximated. It means that for each $\mathbf{C} \in \mathcal{C}_{E}, E(\varphi)(t) \approx E(\mathbf{d})(t)$.

Let $\mathbf{C}^{*}$ stand for constants for which the function $h$ attains a minimum. Then, a satisfactory approximation should have the property that $\mathbf{C}^{*}$ exhibits a weak dependence on $\mathbf{d}_{0}$ and $\mathbf{f}$. This, in turn, is connected with assumed functions $\pi_{T}$ and SDS which reflect the correctness of averaged modelling. Finally, we have to choose a constant $\overline{\mathbf{C}}$ from the set of $\mathbf{C}^{*}$ by an averaging method. Then,

$$
\left.\overline{\mathbf{C}}=A v\left\{\mathbf{C}^{*}: \mathbf{C}^{*}\left(\mathbf{d}_{0}, \overline{\mathbf{f}}\right), \mathbf{d}_{0} \in \overline{\mathcal{M}}, \overline{\mathbf{f}} \in \overline{\mathcal{F}}\right)\right\},
$$

where $A v$ means the averaging operation. The obtained constants $\overline{\mathbf{C}}$ determine a dimensionally reduced dynamical system $\operatorname{RDS}(\overline{\mathbf{C}})$. By means of formulas (14) and (15), an approximation and identification procedure denoted generally by app is established.

We can discuss also another kind of an approximation procedure. Let us assume that there is a relation $\mathcal{R}_{\text {app }}$ between variables and forces of SDS and constants $\mathbf{c}_{\text {EDS }}$ considered in the elementary dynamical system.

Let us consider a system of equations

$$
\begin{aligned}
& \left\{\overline{\mathcal{L}}_{T G}, \bar{T}_{C}\right\}\left(\mathbf{C}, \mathbf{d}_{g}, \mathbf{d}_{c}\right)=\{\overline{\mathbf{f}}, \mathcal{B}\}, \\
& \mathcal{R}_{\mathrm{app}}\left(\mathbf{d}_{g}, \mathbf{d}_{c}, \overline{\mathbf{f}}, \mathcal{B}_{c}, \mathbf{c}_{\mathrm{EDS}}\right)=0 .
\end{aligned}
$$

We assume that the Eq. (16) and the relation $\mathcal{R}_{\text {app }}$ create together such a system of equations which enables to determine $\overline{\mathbf{C}}=\mathbf{C}\left(\mathbf{c}_{\mathrm{EDS}}\right)$. In such a case, the reduced dynamical system is also determined.

Summing up these considerations, let us notice that the following general procedure is established: $\{\mathrm{EDS}, \mathrm{DR}\} \rightarrow \operatorname{RDS}(\overline{\mathbf{C}})$. It means that the dimensional reduction procedure $\mathrm{DR}=\left\{\pi_{T}, \pi_{f T}, \mathrm{SDS}\right.$, app $\}$ acting on an elementary dynamical system (2) leads to obtaining the reduced dynamical system RDS. Consequently, RDS is considered as describing approximately the evolution of our initially introduced physical system.

\subsection{Remarks on the description of the system by various types of dimensional reductions}

Having in mind the complexity of processes in small scale, we have assumed that a general model will contain a set of submodels. Then, during the evolution of the material, we can sequentially apply various submodels when the quality of description undergoes a change. Let us mention the transition of elastic material to slip on a single slip surface. Then, after exceeding a critical condition, a new variable related to slip appears. It has its own evolution equation.

In general, we can expect a considerable number of changes of type of models. When we apply a dimensional reduction procedure in a very formal way, we are aware what kind of approximation of elementary dynamical system is applied. Thereby, we can specify conditions when such an approximation is valid. This leads to the determination of critical conditions for the validity of a given model on basis of the applied dimensional reduction procedure.

It is expected that a future systematic activity in such a field will be possible owing to the context provided by collection of dynamical systems. Then, full modelling of materials will contain corresponding equations together with critical conditions for validity of particular models. 


\section{Continuum with finite-dimensional fields}

\subsection{General formulation}

We have formulated mathematical foundations of continuum mechanics within rational mechanics [1]. We discuss here some generalization of this formulation with special regard to placing it in the more general theory of collection of dynamical systems with dimensional reduction.

Let us note that continuum mechanics describes properties of a material in a more averaged way than this is done by molecular dynamics for instance. However, fields on continuum create predominantly infinitedimensional spaces. On the other hand, molecular dynamics equations have a finite number of degrees of freedom. Thereby, equations of continuum mechanics should be reformulated in order to have a smaller number degree of freedom than the atomistic models have. Only then the continuum theory can be considered as simpler and can be obtained by means of the dimensional reduction procedure.

The idea of introducing finite-dimensional fields consists in the assumption on validity of balance equations not for all subbodies of the body $\mathcal{B}$ but only for their determined family $\mathcal{K}$. It is in fact a generalization of classical formulation since all subbodies create a particular case of $\mathcal{K}$.

The notions of the classical continuum should be modified with respect to a finite family of subbodies $\mathcal{K}$ in order to obtain spaces of finite-dimensional fields. We would like also to connect notions related to continuum with an elementary dynamical system. To this end, mappings defining geometrical objects related to dynamical subsystems will be used. They have been introduced in general assumptions characterizing the collection of dynamical systems.

Consequently, we can consider mappings $G_{x}, G_{L}, G_{S}, G_{V}$ which assign zero, one, two and three-dimensional geometrical objects to each subsystem correspondingly.

Let us apply the special case of the map $G_{V}: \mathcal{M}_{\Pi} \rightarrow\left(2^{E_{e}}\right)^{P}$ as $G_{V}=G_{K}$. Let $\mathcal{M}_{K}=\{\mathcal{K}\}$ be a set of possible families of $\mathcal{K}=\left\{K_{h}\right\}$, where $K_{h} \subset E_{e}$ and int $K_{g} \cap$ int $K_{h}=\emptyset, g, h \in I_{P}$. Then, $G_{K}: \mathcal{M}_{\Pi} \rightarrow \mathcal{M}_{K}, G_{K}\left(\left\{\varphi_{h}\right\}\right)=\mathcal{K}$. We introduce also the mapping $G_{K h}\left(\left\{\varphi_{h}\right\}\right)=K_{h}$.

Consequently, the mapping $G_{K}$ introduces three-dimensional disjoint subsets of $E_{e}$ which will be further interpreted as partition of the body $\mathcal{B}$ into the set of subbodies $K_{h}$ and $\bigcup K_{h}$.

Let us note that $G_{K}\left(\left\{\boldsymbol{\varphi}_{h}\right\}(t)\right)=\left\{K_{h}\right\}(t)$ describe the evolution of $\mathcal{B}(t)=\bigcup_{h} K_{h}(t)$ in time. $K_{h}(t)$ will be also denoted by $\chi_{t}\left(K_{h}\right)$ in what follows.

Definition 1 The body associated with the dynamical system $\dot{\varphi}=L(\boldsymbol{\varphi}, \mathbf{f})$ is defined with the help of mapping $G_{K}$ as $\mathcal{B}_{\varphi}=\bigcup_{h \in I_{P}} K_{h}$.

Let us apply also the function $G_{x}=G_{\chi}$ which assigns a point $\chi_{h}$ as a distinguished point of $\chi\left(K_{h}\right)$ to each subsystem. Thereby, $G_{\chi}: \mathcal{M}_{\Pi} \rightarrow\left\{\left\{\chi_{h}\right\}\right\}$. We introduce also a mapping $\bar{\chi}: \mathcal{M}_{K} \rightarrow\left\{\left\{\chi_{h}\right\}\right\}$. Consequently, $G_{\chi}=\bar{\chi} \circ G_{K}$. In particular $G_{\chi}=G_{I}$ defines $\mathbf{X}_{h}$ as a distinguished point of $K_{h}$ in a reference configuration.

Let $H_{\chi h}=\left\{\chi_{m}, m \in I_{h}^{a}\right\}$ be a particular case of the set $H_{\alpha h}$ defined in the previous section. Then, we introduce the function $a_{h}: H_{\chi h} \rightarrow V_{a h}$ and $a:\left\{\left\{H_{\chi h}\right\}\right\} \rightarrow\left\{\left\{a_{h}\left(\left\{\chi_{m}\right\}\right)\right\}\right\}$ as a function of kinematical dependence between subsystems defined here with the help of family $\mathcal{K}$. $V_{a h}$ is a linear space.

The function $\bar{\chi}$ assigns a set of discrete values of the field $\chi_{h}, h \in I_{P}$ to the body $\mathcal{B}$ with the help of the family $\mathcal{K}$. Similarly, the function $a$ assigns a set of discrete values of the field $a_{h}, h \in I$ to the body. However, $a_{h}$ depends on the finite set of values $\chi_{m}, m \in I_{h}^{a}$. The definition of the finite set is introduced with the help of the set of indexes $I_{h}^{a}$. This set in turn contains indexes of elements of $\mathcal{K}$ which have influence on the value of $a_{h}$. Usually, they will be related to some neighbouring sets $K_{i}$ of $K_{h}$. Thus, the functions $\bar{\chi}$ and $a$ together can express nonlocal properties of the body.

Let $\bar{V}_{D}=\left\{\{\bar{\chi}, a\}:\{\bar{\chi}, a\}=\left\{\chi_{h}, a_{h}\right\}, h \in I_{P}\right\}$. Let us define the space $V_{\kappa}$ of deformation functions $\chi_{\kappa}$ of the body $\mathcal{B}$ with respect to a given configuration $\kappa$ as $V_{\kappa}=\left\{\chi_{\kappa}: \chi_{\kappa}=\lambda \circ \kappa^{-1}, \lambda, \kappa \in \mathcal{C}\right\}$ [1]. Let furthermore, $\alpha_{\chi}: \bar{V}_{D} \rightarrow V_{\kappa}$ be a function and $\chi_{\kappa}^{\mathcal{K}}=\alpha\left(\left\{\chi_{h}, a_{h}\right\}\right), \chi_{\kappa}^{\mathcal{K}}\left(\mathbf{X}_{h}\right)=\chi_{h}$.

Definition 2 The deformation function associated with the distinguished family of subbodies $\mathcal{K}$ is a function $\chi_{\kappa}^{\mathcal{K}}$ of the form $\chi_{\kappa}^{\mathcal{K}}=\alpha_{\chi}\left(\left\{\chi_{h}, a_{h}\right\}\right)$.

Definition 3 The motion of the body $\mathcal{B}$ associated with the family of sets $\mathcal{K}$ is a continuous map $\chi_{t}:[0, T] \rightarrow$ $\left\{\chi_{\kappa}^{\mathcal{K}}\right\}$.

With the help of above definitions, we have obtained deformation function and motion of the body in connection with the elementary dynamical system. This connection is expressed by means of functions $G_{K}$ and $G_{\chi}$. 
Let us notice that by means of functions $a_{h}$, gradients of various degree can be introduced. Consequently, these functions will be important for the determination of constitutive equations.

We also introduce a function $\bar{T}$ on $\mathcal{K}$, which represents temperature, as $\bar{T}: \mathcal{K} \rightarrow R^{P}, \bar{T}\left(\left\{K_{h}\right\}\right)=\left\{T_{h}\right\}$. Let $I_{h}^{b} \subset I_{P}$ and $H_{T h}=\left\{T_{n}, n \in I_{h}^{b}\right\}$. Then, we introduce a function $b_{h}$ by analogy to $a_{h}$ as $b_{h}: H_{T h} \rightarrow V_{b h}$ and $b:\left\{\left\{H_{T h}\right\}\right\} \rightarrow\left\{\left\{b_{h}\left(T_{n}\right)\right\}\right\}$.

Let us note that assignation of the value $T_{h}$ to the point $\chi_{h}$ in $\chi\left(K_{h}\right)$ is not so simple as defining $\chi_{h}$ which has direct geometrical interpretation. At this moment, we do not discuss this problem precisely. Larger context for defining the temperature within continuum mechanics description will be discussed in what follows.

Let $\bar{V}_{\mathrm{TM}}=\left\{\{\bar{T}, b\}:\{\bar{T}, b\}=\left\{T_{h}, b_{h}\right\}, h \in I_{P}\right\}, V_{\mathrm{TM}}=\{T(\mathbf{x}): \mathbf{x} \in \chi(\mathcal{B})\}$. Let us consider a function $\alpha_{T}: \bar{V}_{\mathrm{TM}} \rightarrow V_{\mathrm{TM}}$ and $T^{\mathcal{K}}=\alpha_{T}\left(\left\{T_{h}, b_{h}\right\}\right)$.

Definition 4 The temperature field $T^{\mathcal{K}}$ associated with the distinguished family of subbodies $\mathcal{K}$ is the field obtained with the help of the function $\alpha_{T}$ as $T^{\mathcal{K}}=\alpha_{T}\left(\left\{T_{h}, b_{h}\right\}\right)$.

The function $\alpha_{\chi}$ assigns a deformation function field $\chi_{\kappa}^{\mathcal{K}}$ to the set of its discrete values. The aim of this function is to introduce a continuous field $\chi$ on the body $\mathcal{B}$. A similar role plays the function $\alpha_{T}$ for $T$. Thus, the spaces of such fields $\operatorname{Im} \alpha_{\chi} \subset V_{\kappa}$ and $\operatorname{Im} \alpha_{T} \subset V_{\mathrm{TM}}$ are finite-dimensional, where $\operatorname{Im} \phi$ means the image of a function $\phi$. Consequently, we have obtained a continuum with finite-dimensional fields in relation to an elementary dynamical system.

\subsection{Balance of mass and energy for continuum mechanics}

In the previous section, we have introduced assumptions related to the collection of dynamical systems. They admit the existence of functions $\tilde{m}, J_{\varphi i j}, c_{\varphi}, E_{\varphi}, W_{\varphi i j}, R_{\varphi}$ which introduce masses $m_{i}$, efflux of mass $J_{i j}$, source of mass $c_{i}$, efflux of energy $W_{i j}$ and source of energy $R_{i}$ accompanied by subsystems. Consequently, the mentioned functions indexed here by $\varphi$ are referred directly to the elementary dynamical system.

We use these functions to the reformulation of the general form of balance of mass and energy equations defined for collection of dynamical systems to the case of continuum.

Let $\tilde{m}: \mathcal{M}_{\Pi} \rightarrow\left\{\left\{m_{h}\right\}\right\}$ be a mapping which determines a set of masses related to collection of dynamical systems. Let $\mathcal{M}_{M}=\left\{\left\{M_{h}\right\}\right\}$ and $M: \mathcal{M}_{K} \rightarrow \mathcal{M}_{M}$ be a function which determines masses assigned to $K_{h}$. We have also that $\pi_{h} \circ M(\mathcal{K})=M_{h}$, where $M_{h}$ is the total mass related to $K_{h} . M_{h}$ are defined by means of the relation $M \circ G_{K}=i \circ \tilde{m}$, where $i$ is identity mapping. Consequently, a system of masses related to continuum is introduced by means of mapping $\tilde{m}$ defined on an elementary dynamical system. Let $\mathcal{B}=\bigcup_{h} K_{h}, h \in I_{B}$, where $I_{B} \subset I_{P}$ is a set of indexes defining an arbitrary subbody $\mathcal{B}$ of the body also denoted by $\mathcal{B}$. Then, $M(\mathcal{B})=\sum_{h \in I_{B}} M_{h}$. Using this definition, we obtain the mass related to subbodies as a kind of measure defined on the body.

The energy accompanied by the elementary dynamical system is introduced by means of the function $E_{\varphi}: \mathcal{M}_{\Pi} \rightarrow R^{P}$. Then, energy $\mathcal{E}: \mathcal{M}_{K} \rightarrow\left\{\mathcal{E}_{h}\right\}, \mathcal{E}_{h}=\pi_{h} \circ \mathcal{E}\left(\left\{K_{h}\right\}\right)$ assigned to each $K_{h}$, is defined by means of the relation $\mathcal{E} \circ G_{K}=i \circ E_{\varphi}$. As a result, we are able to define the energy related to the subbody as $\mathcal{E}(\mathcal{B})=\sum_{h} \mathcal{E}_{h}$. We assume further that $\mathcal{E}=E+\mathcal{T}$ is the sum of internal energy and kinetic energy.

Source of mass $c_{\varphi}: \mathcal{M}_{\Pi} \rightarrow R^{P}$ and source of energy $R_{\varphi}: \mathcal{M}_{\Pi} \rightarrow R^{P}$ are defined now as $C: \mathcal{M}_{K} \rightarrow$ $R^{P}, R: \mathcal{M}_{K} \rightarrow R^{P}$ by means of relations $C \circ G_{K}=i \circ c_{\varphi}$ and $R \circ G_{K}=i \circ R_{\varphi}$. These quantities are defined for subbodies by means of expressions $C(\mathcal{B})=\sum_{h} C_{h}, R(\mathcal{B})=\sum_{h} R_{h}$.

Efflux of mass $J_{\varphi i j}: \mathcal{M}_{\Pi} \rightarrow R$ and efflux of energy $W_{\varphi i j}: \mathcal{M}_{\Pi} \rightarrow R$ are defined as $J_{i j}: K_{i} \times K_{j} \rightarrow R$, where $J_{i j}$ is determined by means of $J_{i j} \circ\left(G_{K i} \times G_{K j}\right)=i \circ J_{\varphi i j}$ and $W_{i j}: K_{i} \times K_{j} \rightarrow R$, where we obtain $W_{i j}$ from $W_{i j} \circ\left(G_{K i} \times G_{K j}\right)=i \circ W_{\varphi i j}$.

Let us consider the boundary of the body $\partial \mathcal{B}=\partial \bigcup_{h} K_{h}$. Then, $J(\partial \mathcal{B})=\sum_{i \in I_{B}, m \in I_{P}-I_{B}} J_{i m}$ and $W(\partial \mathcal{B})=\sum_{i \in I_{B}, m \in I_{P}-I_{B}} W_{i m}$.

We consider also $\partial \mathcal{B}_{s} \subset \partial \mathcal{B}$ which is defined as $\partial \mathcal{B}_{s}=\bigcup_{h \in I_{s}}\left(\partial K_{h} \cap \partial \mathcal{B}\right), I_{s} \subset I_{B}$. There exists a relation between $\partial \mathcal{B}_{s}$ and the set of $J_{i m}$. We assume that a pair of indexes $\{i, m\}$ is associated with $\partial \mathcal{B}_{s}$ if $\partial \mathcal{B}_{s}$ is a border between subsystems $i$ and $m$. Then, $J\left(\partial \mathcal{B}_{s}\right)=\sum_{i \in I_{s}, m \in I_{s i}} J_{i m}$.

With the help of the introduced functions, the balance of mass Eq. (4) interpreted in terms of continuum is given by

$$
\dot{M}(\mathcal{B})+J(\partial \mathcal{B})-C(\mathcal{B})=0 .
$$


with the additional condition satisfied for arbitrary $\partial \mathcal{B}_{s} \subset \partial \mathcal{B}$

$$
J\left(\partial \mathcal{B}_{s}\right)=\bar{J}\left(\partial \mathcal{B}_{s}\right) .
$$

The balance of energy Eq. (7) expressed in terms of continuum is assumed in the following form:

$$
\dot{E}(\mathcal{B})+\dot{\mathcal{T}}(\mathcal{B})+W(\partial \mathcal{B})-R(\mathcal{B})=0,
$$

with the additional condition satisfied for arbitrary $\partial \mathcal{B}_{s} \subset \partial \mathcal{B}$

$$
W\left(\partial \mathcal{B}_{S}\right)=\bar{W}\left(\partial \mathcal{B}_{S}\right) .
$$

Neglecting at the moment detailed representations of the below introduced quantities, we formulate also the second law of thermodynamics as a supplementary postulate. This is given with the help of the balance entropy expressed as

$$
P(\mathcal{B})=\dot{S}(\mathcal{B})+H(\partial \mathcal{B})-N(\mathcal{B}) \geq 0
$$

where $S$ is entropy, $H$ is efflux of entropy, and $N$ stands for source of entropy. $P$ represents production of this quantity.

When we use particular representations of quantities which appear in (18)-(22), then we obtain a continuum skeletal dynamical system appropriate for carrying out a dimensional reduction procedure applied to the previously considered elementary dynamical system.

We consider here balance of mass and energy equations only in a similar way as in general considerations for collection of dynamical systems discussed previously. Balance of momentum and balance of angular momentum equations are seen here as laws which are consequences of the balance of mass and energy equations. However they are sometimes postulated independently. This is done especially in continuum mechanics. Such a step is admissible also in the above considerations. However, the decision whether we should consider such laws independently should follow from the particular method of modelling.

\subsection{A particular type of the dimensional reduction procedure}

Physical systems frequently can be characterized by variables of two types. The first one is related to slowly varying and the second one to quickly varying processes. A well-known example is related to a system with deformation and temperature considered within continuum mechanics.

Having this in mind, we illustrate here a general concept of creating foundations for continuum mechanics basing on such a decomposition. Consequently, let us introduce a special case of $\pi_{T}=\left\{\pi_{S T}, \pi_{Q T}\right\}$ in which two parts related to slowly and quickly varying variables are discriminated. As a result of this $\mathbf{d}(t)=\pi_{T}(\varphi(t))$ can be expressed as $\mathbf{d}=\left\{\mathbf{d}_{S}, \mathbf{d}_{Q}\right\}=\left\{\pi_{S T}(\boldsymbol{\varphi}), \pi_{Q T}(\boldsymbol{\varphi})\right\}$.

Let us assume also that the elementary dynamical system is a Hamiltonian dynamical system which takes the form

$$
\begin{aligned}
\frac{\mathrm{d} \mathbf{q}_{i}}{\mathrm{~d} t} & =\mathbf{v}_{i}, \\
m_{i} \frac{\mathrm{d} \mathbf{v}_{i}}{\mathrm{~d} t} & =-\frac{\partial H}{\partial \mathbf{q}_{i}}+\mathbf{f}_{i}
\end{aligned}
$$

where $i \in I_{N}=\{1,2, \ldots, N\}, H$ is a Hamiltonian and $\mathbf{f}_{i}$ is a force acting on $i$ th material point. Let $\mathbf{q}=\left\{\mathbf{q}_{i}\right\}, \mathbf{v}=\left\{\mathbf{v}_{i}\right\}$. Then, variables of our $E D S$ take the form $\boldsymbol{\varphi}=\{\mathbf{q}, \mathbf{v}\}$.

Such an option of the elementary dynamical system suggests the possibility of application of molecular dynamics calculations for creation of physical foundations for continuum model. Consequently, we assume in general that physical foundations for more averaged models, in particular continuum models, are better expressible in smaller scale. This assumption seems to be natural since fundamental physics is related directly to elementary particles and atoms. On the other hand, currently applied equations of more averaged continuum models are based usually on assumptions related to considerable averaged processes. However, this averaging is frequently based on intuition related to corresponding scale or experimental data related to averaged processes. Then, we are not aware which processes are lost during this averaging by intuition. All this justifies the general tendency to obtain better physical foundations following from atomistic models. 
Let us take a set of time instants $t_{0}<t_{1}<\cdots<t_{K}$ which belong to the time interval $T=\left[t_{0}, t_{0}+T\right], t_{K}=$ $t_{0}+T$, and let $I_{K}=\{0,1, \ldots, K\}$. By means of these instants, we divide the time interval into the sum $T=\bigcup_{k} T_{k}, T_{k}=\left[t_{k-1}, t_{k}\right], k=1, \ldots, K$. Then, for each $k$, we can calculate the value of $\tilde{\mathbf{q}}_{k}$ as

$$
\tilde{\mathbf{q}}_{k}=\frac{1}{T_{k}} \int_{T_{k}} \mathbf{q}(t) \mathrm{d} t
$$

A value $\tilde{\mathbf{q}}_{0}=\mathbf{q}\left(t_{0}\right)$ is assigned to $k=0$. With the aid of the sequence of values $\left\{\tilde{\mathbf{q}}_{k}\right\}, k \in I_{K}$, we can generate a function $\tilde{\mathbf{q}}(t)=I_{q}\left(\left\{\tilde{\mathbf{q}}_{k}\right\}\right)$, where $I_{q}$ is an approximation procedure. Now, we are able to decompose the variable $\mathbf{q}(t)$ into two summands,

$$
\mathbf{q}(t)=\tilde{\mathbf{q}}(t)+\delta \mathbf{q}(t)
$$

Thus, $\tilde{\mathbf{q}}(t)$ represents the slowly varying part of $\mathbf{q}(t)$, and $\delta \mathbf{q}(t)$ its rapidly varying part.

The question is which properties should the approximation procedure $I_{q}$ have in order to describe real slowly varying process in accordance with the introduced set $\left\{\mathbf{q}_{k}\right\}$. A slowly varying variable should gradually change its values between given points. This fact should be expressed by possible small values of the second time derivatives. Consequently, the approximation procedure $I_{q}$ could be defined as a process of finding a function $\tilde{\mathbf{q}}(t)$ which satisfy the following set of properties:

$$
\left\{\begin{array}{l}
I_{q}\left(\left\{\tilde{\mathbf{q}}_{k}\right\}\right)=\tilde{\mathbf{q}}(t), \\
\sup _{t \in T}\left(\left|\frac{\partial^{2} \tilde{\mathbf{q}}}{\partial t^{2}}\right|\right) \rightarrow \inf \\
\tilde{\mathbf{q}}\left(\tau_{k}\right)=\tilde{\mathbf{q}}_{k}, \quad \tau_{k}=0.5\left(t_{k-1}+t_{k}\right), \\
\tilde{\mathbf{q}}(t) \in C^{n}, \quad n \geq 2, t \in T .
\end{array}\right.
$$

It should be expected also that $\int_{T_{k}}(\mathbf{q}-\tilde{\mathbf{q}}) \mathrm{d} t=\int_{T_{k}} \delta \mathbf{q} \mathrm{d} t \approx 0$, where " $\approx$ " means equality with an admissible error. As a result of this decomposition, we are able to consider $\dot{\mathbf{q}}(t)=\dot{\tilde{\mathbf{q}}}(t)+\delta \dot{\mathbf{q}}(t)$. Decomposition defined by (26) has been realized by means of a finite element approximation in [11].

Let $A_{t}(\{\mathbf{q}, \dot{\mathbf{q}}\}(t))=\{\tilde{\mathbf{q}}, \delta \mathbf{q}, \dot{\tilde{\mathbf{q}}}, \delta \dot{\mathbf{q}}\}(t)$. Let us introduce further special kinds of variables. Let they be denoted by $\mathbf{d}=\left\{\mathbf{d}_{S}, \mathbf{d}_{Q}\right\}, \mathbf{d}_{S}=\left\{\mathbf{d}_{S C}, \mathbf{d}_{S \xi}, \mathbf{d}_{S V}\right\}$ and $\mathbf{d}_{Q}=\left\{\mathbf{d}_{Q C}, \mathbf{d}_{Q \theta}, \mathbf{d}_{Q V}\right\}$, where $\mathbf{d}_{S C}, \mathbf{d}_{Q C}$ are called configurational variables, $\mathbf{d}_{S V}=\dot{\mathbf{d}}_{S C}, \mathbf{d}_{Q V}=\dot{\mathbf{d}}_{Q C}$. It is admitted that not all $\dot{\mathbf{d}}_{S C}, \dot{\mathbf{d}}_{Q C}$ are present in the set of variables $\mathbf{d}_{S}, \mathbf{d}_{Q}$. Some of them can be neglected. $\mathbf{d}_{S \xi}, \mathbf{d}_{Q \theta}$ are internal state variables.

Taking into account the above decompositions, we assume that there exist mappings $\pi_{S}$ and $\pi_{Q}$ which give $\left\{\mathbf{d}_{S C}, \mathbf{d}_{S \xi}\right\}=\pi_{S} \circ A_{t}(\varphi(t))$ and $\left\{\mathbf{d}_{Q C}, \mathbf{d}_{Q \theta}\right\}=\pi_{Q} \circ A_{t}(\varphi(t))$. Let $D_{S t}\left(\left\{\mathbf{d}_{S C}, \mathbf{d}_{S \xi}\right\}\right)=\mathbf{d}_{S}$ and $D_{Q t}\left(\left\{\mathbf{d}_{Q C}, \mathbf{d}_{Q \theta}\right\}\right)=\mathbf{d}_{Q}$. Then, the form of $\pi_{T}$ is determined by

$$
\pi_{T}=\left\{\pi_{S T}, \pi_{Q T}\right\}=\left\{D_{S t} \circ \pi_{S} \circ A_{t}, D_{Q t} \circ \pi_{Q} \circ A_{t}\right\} .
$$

We introduce also the map $\pi\left(\boldsymbol{\varphi}_{0}\right)=\mathbf{d}_{0}$ which depends on $\pi_{T}$ by assumption that $\pi\left(\varphi\left(t_{0}\right)\right)=\pi\left(\pi_{T}(\varphi(t))\right.$, $\left.\varphi\left(t_{0}\right)=\varphi_{0}\right)$. By means of $\pi$, initial conditions can be transferred between scales.

A similar $S Q$-decomposition can be carried out for forces $\mathbf{f}$. To this end, we calculate the following time-averaged quantities:

$$
\tilde{\mathbf{f}}_{k}=\frac{1}{T_{k}} \int_{T_{k}} \mathbf{f}(t) \mathrm{d} t
$$

Then, we have $\tilde{\mathbf{f}}(t)=I_{f}\left(\tilde{\mathbf{f}}_{k}\right)$ with the help of an approximation procedure $I_{f}$. Finally, we obtain the decomposition

$$
\mathbf{f}(t)=\tilde{\mathbf{f}}(t)+\delta \mathbf{f}(t) .
$$

Let $B_{t}(\mathbf{f}(t))=\{\tilde{\mathbf{f}}(t), \delta \mathbf{f}(t)\}$ and $\pi_{S f} \circ B_{t}(\mathbf{f}(t))=\overline{\mathbf{f}}_{S}, \pi_{Q f} \circ B_{t}(\mathbf{f}(t))=\overline{\mathbf{f}}_{Q}$. Finally, we obtain the general form of the map

$$
\pi_{f T}=\left\{\pi_{S f} \circ B_{t}, \pi_{Q f} \circ B_{t}\right\}
$$


In general, it is expected that mappings $\pi_{T}$ and $\pi_{f T}$ are not entirely independent. Structure of $\pi_{T}$ and operator $\mathcal{L}_{T}$ should have influence on the kind of dimensional reduction applied to $\mathbf{f}$. Establishing possible dependence between $\pi_{T}, \pi_{f T}$ is very complicated in the general case. We postulate merely that there exist relations $\mathcal{R}_{L}\left(\pi_{T}, \pi_{f T}\right)$ which join the considered two mappings. Consequently, it is assumed that in the case of $S Q$ decomposition $\mathcal{R}_{L}=I$, or in other words, the same procedures are represented by $\pi_{T}$ and $\pi_{f T}$.

Accordingly, we have obtained the following decomposition of variables of the new dynamical system $\mathbf{d}=\left\{\mathbf{d}_{S}, \mathbf{d}_{Q}\right\}=\left\{\mathbf{d}_{S C}, \mathbf{d}_{S \xi}, \mathbf{d}_{S V}, \mathbf{d}_{Q C}, \mathbf{d}_{Q \theta}, \mathbf{d}_{Q V}\right\}$.

With the help of $S Q$-decomposition of variables represented by mappings $\pi_{T}, \pi_{f T}$, we would like to obtain premises for the determination of more detailed forms of Eqs. (12) and (13). However, we do not discuss here any particular models.

The above discussion accentuates the difference in this approach and statistical mechanics approach where a physical basis for continuum mechanics can also be created. In our case, all models are deterministic. The method of averaging of solutions defines temperature for instance as related to averaging of quickly varying processes. Thereby, we see that a precise definition of temperature on this way can be difficult but is important. This is not stochastic averaging.

Let us note that temperature should be well defined also in case of deterministic models. Stochasticity in this case is related rather to initial conditions. However, it is imaginable that we know such initial conditions precisely. Then, temperature should be also well defined. Thereby, stochasticity should not be a necessary condition for the definition of temperature. Let us also note that a formulation of stochastic approach in case of a multiscale method represented by collection of dynamical systems has been discussed in [12].

We have already introduced two kinds of variables $\chi_{h}$ and $T_{h}$ for our continuum with finite-dimensional fields. It means that $S Q$-decomposition is accepted in continuum description. Indeed, $T_{h}$ represents averaged properties of quickly varying processes and $\chi_{h}$ the slowly varying ones.

We can introduce the following interpretation of the introduced variables $\mathbf{d}_{S C}=\left\{\chi_{h}\right\}, \mathbf{d}_{S V}=\left\{\dot{\chi}_{h}\right\}, \mathbf{d}_{S \xi}=$ $\left\{\eta_{h}\right\}$ as internal state variables, $\mathbf{d}_{Q C}=\left\{T_{h}\right\}, \mathbf{d}_{Q \theta}=\emptyset, \mathbf{d}_{Q V}=\emptyset$. In the two last cases, we have neglected internal state variables related to quickly varying processes and time derivative of temperature $T_{h}$.

It is possible to deduce using also the form of EDS (23) that the balance of energy Eq. (20) can be expressed in the form [9]

$$
\dot{E}(\mathcal{B})+\dot{\mathcal{T}}(\mathcal{B})+W_{S}(\partial \mathcal{B})+W_{Q}(\partial \mathcal{B})-R_{S}(\mathcal{B})-R_{Q}(\mathcal{B})=0 .
$$

We can assume also that the balance of mass equation subsequently applied takes the simple form $\dot{M}_{h}=0$.

The above equations lead to the determination of the continuum skeletal dynamical system by means of application of particular representation of quantities in (31).

\section{Nanoscale models}

\subsection{Importance of nanoscale models}

The above discussed multiscale method of modelling provides a theoretical framework for the formulation of various models in a unified way. Furthermore, the aim of this method is to provide convenient conditions for transition from atomic scale to more averaged continuum models within one theoretical scheme.

As it was discussed previously, this transition is not easy since many physical processes at intermediate scales can be continued. In order to overcome this obstacle, we should distinguish a particular scale which corresponds to a considerable set of such processes. We estimate that nanoscale models are appropriate for being a bridge between atomistic models and more applicable continuum ones. This is so since many mechanisms especially related to inelastic deformation are associated with nanoscale.

Consequently, we should elaborate a methodology in construction of nanoscale models. Two important problems appear then. We have to do with some models of martensitic transformation for instance which could be considered as nanoscale $[13,14]$. They are based on Landau theory. In such a case, the free energy is expressed by means of polynomes [15]. This is not too convenient for modelling of larger class of phenomena. High degrees of polynomes which appear when modelled phenomena become more complex make the corresponding description difficult to formulation and interpretation. Consequently, methods of defining of the free energy function taking into account the complex geometrical shape of the graph of this function is the first problem.

We encounter at the nanoscale level phenomena in material leading to qualitatively different models. Let us mention martensitic transformation and slip plasticity for instance. They represent two different mechanisms 
of inelastic deformation. Transition and interaction between them is very important in case when we elaborate a more averaged model basing on nanoscale description. Thereby, we should solve additionally the problem of unification of description considered within the same nanoscale approach. It seems that such a task could be assigned to form the free energy.

Having in mind the above-discussed aspects of modelling, we would like to illustrate in the next subsections a methodology of construction of nanoscale models taking as an example conjugation of martensitic transformation and slip plasticity.

\subsection{Kinematical considerations}

We try to introduce the methodology of nanoscale modelling within mechanics of materials. We should do it also in the context of whole unified mechanics of materials presented in this paper. On the one hand, we should place this model within collection of dynamical systems. On the other hand, nanoscale modelling is sufficiently complex as such, and we should devote special attention especially to the methodology of constructing the free energy function. Then, joining of both tasks would be too complicated and perhaps unclear. In order to avoid such complications, we discuss concisely the context provided by collection of dynamical systems in order to show where within this context further considerations on nanoscale modelling are placed.

We should distinguish first collection of dynamical systems for our description. We are going to consider material as continuum for nanoscale modelling. Then, we can distinguish dynamical subsystems directly by geometrical objects $K_{h}$ which create the continuum and by variables determined on $K_{h}$ and corresponding to processes which happen in this scale. Consequently, the size of $K_{h}$ and corresponding variables are chosen in order to see particular important processes for the described phenomena.

The free energy within this context is a scalar quantity $F_{h}$ assigned to the $h$ th set $K_{h}$. However, relations of $F_{h}$ with other sets $K_{h^{\prime}}$ are introduced by gradients of variables represented by functions $a_{h}$ discussed in introduction of a continuum with finite-dimensional fields.

In this section, we do not apply directly subsystems in order to concentrate our attention on methodology of constructing the free energy. Thereby, our free energy will be understood here as function assigned to a point $\mathbf{X}$ of the continuum as $F(\mathbf{X})$. Elaborated within the presented below methodology, the free energy function $F(\mathbf{X})$ can be next adapted to finite-dimensional description within collection of dynamical systems. Then, we obtain $F_{h}$ from $F(\mathbf{X})$ and application of finite-dimensional fields. Let us note that by incorporation of the free energy into balance of energy equations we can construct in fact a skeletal dynamical system (SDS) corresponding to nanoscale model which can be next supported by an atomistic model. We can interpret also the nanoscale finite-dimensional model as an elementary dynamical system (EDS) and apply it to the production of more averaged models.

The scale of averaging assumed in the description presented here will be manifested by types of variables introduced in relation to objects modelled. Complexity of form of the free energy also manifests the scale of averaging by the fact which phenomena are described by the model. The formal approach expressed by the precise definition of the structure of dynamical subsystems and its geometrical characteristics will be important in future when formal numerical simulations should be carried out.

We consider here various kinematical aspects related to martensitic transformation and slip plasticity. The whole description presented will not be complete. We accentuate rather a methodology here. Our discussion is carried out taking into account properties of $\mathrm{CuAl}$ alloy as motivation for the description which has representative properties for a large class of materials undergoing martensitic transformation.

The dominant feature which characterizes the martensitic transformation is a shear strain [16]. The martensitic transformation is initiated when a critical shear stress is exceeded. Let us note that models describing martensitic transformation can describe an averaged martensitic structure. However, a nanoscale model has to see separate martensite variants $[17,18]$.

In order to distinguish the characteristic shear strain corresponding to a given martensite variant, shear systems are introduced. Let us consider first material in austenitic state. The $I$ th shear system is represented by a pair of orthonormal vectors $\mathbf{d}_{I \mu}, \mu=1,2, I \in I_{S A} . I_{S A}$ consists of all indexes which determine all distinguished shear systems related to austenite.

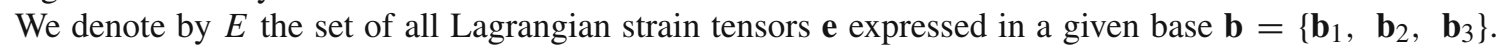
Measure of the shear strain in the $I$ th shear system is given by

$$
e_{I 12}=\mathbf{d}_{I 1} \mathbf{e d}_{I 2} .
$$


Directions of micrononhomogeneous shear for this structure are defined by $\mathbf{d}_{I \mu}$, where $I=(i j), \mu=1,2$. ( $i j$ ) define the plane in which the shear takes place. This can be expressed by means of $\mathbf{b}_{i}, \mathbf{b}_{j}$ in which the shear takes place. The vectors $\mathbf{d}_{I \alpha}$ are defined by formula $\mathbf{d}_{(i j) \mu}=\frac{\sqrt{2}}{2}\left(\mathbf{b}_{i}+(-1)^{\mu} \mathbf{b}_{j}\right)$ for $C u A l$ alloy for instance.

We have introduced a system of multi-indices. The first one is $I=(i j)$, next ones are $J=(I, \mu)$ and $K=(J, v)=(I, \mu, v) . I$ is defined by $i, j=1,2,3, i \neq j, \mu, v=1,2$. The set $I_{K}$ contains all indexes $K$. With the help of such indexes, all martensite variants are classified. In particular, the role of some additional variables characterizing nonhomogeneous deformation related to index $\mu$ and internal rotations related to index $v$ is accentuated.

Let us notice that a critical parameter for the initiation of slip is also a shear strain. Therefore, we introduce additionally slip systems determined by a pair of unit and orthogonal vectors $\left\{\mathbf{m}_{\alpha}, \mathbf{n}_{\alpha}\right\}, \alpha \in I_{S L}$. Then, the shear strain related to the slip system is given by

$$
e_{\alpha m n}=\mathbf{m}_{\alpha} \mathbf{e n}_{\alpha} .
$$

Difference between shear system and slip system rests on the interpretation of phenomena after exceeding a critical strain. The martensite appears in $I$ th shear system after exceeding a critical shear strain $e_{I 12}^{*}$, and the strain $e_{I 12}$ is further developed. The strain $e_{\alpha m n}$ is determined in an elastic region only. After exceeding a critical strain, the situation changes qualitatively since slip appears. Slip on a slip surface is connected with displacements defined on this surface of discontinuity. The mentioned displacements are an entirely different kind of deformation measure. The measure of strain $e_{\alpha m n}$ is not interpreted in $\left\{\mathbf{m}_{\alpha}, \mathbf{n}_{\alpha}\right\}$ slip system beyond the elastic region of austenite.

The elastic domain for austenite is expressed as a subset $A \subset E$. Furthermore, we discuss the elastic domain of martensite $M_{K} \subset E$ and the spinodal region $S \subset E$. If $\mathbf{e}$ attains $\partial A$, then the situation changes qualitatively. The tensor $\mathbf{e}$ is not considered as full measure of deformation. Next, components have to be adjoined.

Taking into account properties of $C u A l$ alloy, we introduce nonhomogeneous deformation related to nanoscale by means of vectors $\boldsymbol{\xi}_{\lambda}$ representing some shifts of atoms. Thus, we have

$$
\mathbf{y}_{\lambda}=\mathbf{x}+\boldsymbol{\xi}_{\lambda}
$$

which reflect multicomponent properties of the material. Such a family of deformation functions can be next approximated by one deformation function and relative displacement vectors.

The internal rotations are introduced by variables

$$
\mathbf{a}_{v}=\mathbf{R}^{-1}(\mathbf{F}-\mathbf{R}) \mathbf{p}_{v}=\left(\mathbf{R}^{-1} \mathbf{F}-\mathbf{1}\right) \mathbf{p}_{v}
$$

where $\mathbf{p}_{v}$ represents pairs of vectors defining the position of interfaces, and $\mathbf{F}$ is the gradient of deformation.

The total domain is considered as $D=A \cup S \cup \bigcup_{K} M_{K} \subset E$. The set $S$ plays a special role. Its boundary $\partial S$ determines critical conditions for the initiation of martensitic transformation or slip. Thus, we distinguish some subsets $\partial S=\partial S_{A} \cup \bigcup_{K} \partial S_{K}$, where $\partial S_{A}=\partial A, \partial S_{K}=\partial M_{K}$. Furthermore, $\partial S_{A}$ consists of the following parts:

$$
\partial S_{A}=\bigcup_{I} \partial S_{I} \cup \bigcup_{\alpha} \partial S_{\alpha}
$$

$\partial S_{I}$ is a criterion of $I$ th kind of martensite initiation, and the form of $\partial S_{\alpha}$ is responsible for the beginning of slip in the $\alpha$ th slip system. Similarly, we introduce also a partition

$$
\partial S_{K}=\bigcup_{K^{\prime}} \partial S_{K K^{\prime}} \cup \partial S_{K A} \cup \bigcup_{\beta} \partial S_{K \beta}
$$

where $\partial S_{K K^{\prime}}$ determines conditions for initiation of transformation of $K$ th into $K^{\prime}$ th martensite variant, $\partial S_{K} A$ determines initiation of $K$ th martensite variant into austenite, and $\partial S_{K \beta}$ initiation of slip in $\beta$-slip system determined in $K$ th martensite variant.

At this moment, we consider also additional elastic regions $M_{K}$. As a result, we have to define slip systems in this kind of domain.

Let $\mathbf{e}_{K}=\frac{1}{2}\left(\mathbf{F}_{K}^{T} \mathbf{F}_{K}-\mathbf{1}\right)$ stand for the strain tensor corresponding to equilibrium of the $K$ th variant of martensite. The strain tensor $\mathbf{e}$ is defined with respect to undeformed austenite configuration. Slip systems in 
the $K$ th martensite variant are determined by means of strain $\tilde{\mathbf{e}}_{K}=\frac{1}{2}\left(\tilde{\mathbf{F}}_{K}^{T} \tilde{\mathbf{F}}_{K}-\mathbf{1}\right)$ defined with respect to undeformed martensite which corresponds to the gradient of deformation $\tilde{\mathbf{F}}_{K}$.

On the other hand, we would like to consider a common strain measure $\mathbf{e}$ on the whole domain. The gradient of deformation can be expressed as $\mathbf{F}=\tilde{\mathbf{F}}_{K} \mathbf{F}_{K}$. With the help of this we obtain

$$
\begin{aligned}
\mathbf{e} & =\frac{1}{2}\left(\mathbf{F}^{T} \mathbf{F}-\mathbf{1}\right)=\frac{1}{2}\left(\mathbf{F}_{K}^{T} \tilde{\mathbf{F}}_{K}^{T} \tilde{\mathbf{F}}_{K} \mathbf{F}_{K}-\mathbf{1}\right) \\
& =\mathbf{F}_{K}^{T} \frac{1}{2}\left(\tilde{\mathbf{F}}_{K}^{T} \tilde{\mathbf{F}}_{K}-\mathbf{1}\right) \mathbf{F}_{K}+\frac{1}{2}\left(\mathbf{F}_{K}^{T} \mathbf{F}_{K}-\mathbf{1}\right)=\mathbf{F}_{K}^{T} \tilde{\mathbf{e}}_{K} \mathbf{F}_{K}+\mathbf{e}_{K} .
\end{aligned}
$$

Consequently,

$$
\tilde{\mathbf{e}}_{K}=\left(\mathbf{F}_{K}^{T}\right)^{-1}\left(\mathbf{e}-\mathbf{e}_{K}\right) \mathbf{F}_{K}^{-1}
$$

expresses $\tilde{\mathbf{e}}_{K}$ with the help of known $\mathbf{e}_{K}$ and varying $\mathbf{e}$.

Let $\left\{\mathbf{m}_{K \beta}, \mathbf{n}_{K \beta}\right\}, \beta \in I_{S L K}$ represent the set of slip systems defined in $M_{K}$. With the help of (39), we define shear strain in the $\beta$ th slip system as

$$
e_{K \beta m n}=\mathbf{m}_{K \beta}\left(\left(\mathbf{F}_{K}^{T}\right)^{-1}\left(\mathbf{e}-\mathbf{e}_{K}\right) \mathbf{F}_{K}^{-1}\right) \mathbf{n}_{K \beta} .
$$

Thus, we are able to express a critical strain condition for slip initiation with the help of (40). Then, $\partial S_{K \beta}$ can be determined.

The set $\partial S_{K A}$ can be defined with the help of the shear system introduced for austenite. Transformation of austenite in the $I$ th shear system leads to $K$ th martensite variant, where $I \subset K$. However, for given $I$, we have four martensite variants. They are connected with internal rotations $\mathbf{R}_{K}$ which in turn depend on relative displacement vectors.

The set $\partial S_{K K^{\prime}}$ cannot be expressed with the help of the one shear system. Therefore, a more general situation should be considered. Let us notice that the shear system generates a one-dimensional path parameterized by $e_{I 12}$ in $E$. We could generalize this fact into a more general one-dimensional path.

Consequently, let us consider a one-dimensional path $G\left(K, K^{\prime}\right) \subset E$ which joins equilibrium states of $K$ th and $K^{\prime}$ th martensite variants. Then, the critical condition related to $\partial S_{K K^{\prime}}$ would be defined by means of $G\left(K, K^{\prime}\right)$.

The measure of slip deformation is connected with a discontinuity of deformation function $\chi$ on the slip surface. The slip surface $\mathcal{S}$ can be considered as composed of two material surfaces $\mathcal{S}^{+}, \mathcal{S}^{-}$associated with two parts of the body. They are in contact on $\mathcal{S}$. This follows from that two deformation functions $\chi^{+}$and $\chi^{-}$ related to parts of the body on the "+" and on the "-" sides of $\mathcal{S}$ are considered.

The measure of slip on the slip surface is considered by means of slip displacement tangent to the slip surface when they are small [19] and defined by

$$
\mathbf{u}_{S}\left(\mathbf{x}_{S}^{-}(t+s)\right)=\chi^{+}\left(\mathbf{X}^{-}, t+s\right)-\chi^{-}\left(\mathbf{X}^{-}, t+s\right) .
$$

We have furthermore the property

$$
\frac{\mathrm{d} \mathbf{u}_{S}}{\mathrm{~d} t}=\mathbf{v}^{+}\left(\mathbf{X}^{-}, t\right)-\mathbf{v}^{-}\left(\mathbf{X}^{-}, t\right)=\mathbf{s}\left(\mathbf{x}_{S}\right)
$$

which defines velocity of slip in the point $\mathbf{x}_{S}$ of the surface.

Summarizing, we see that nanoscale description should have at its disposal an appropriate number of variables which enable us to see by the model phenomena corresponding to this scale. In this case, we have to do with separate martensite variants and corresponding separate interfaces, slip surfaces and dynamical processes associated with these objects. 


\subsection{Free energy}

In this subsection, we discuss the methodology of defining the free energy. This is done by considering the graph of this function as fibre bundle spanned on characteristic geometrical elements having physical interpretation.

The here discussed model of plasticity is characterized by slip surfaces $\mathcal{S}$. Discontinuity of deformation function is the main feature related to these surfaces. The three-dimensional Lebesgue measure $\mu\left(\bigcup_{\mathcal{B}}(\mathcal{S})\right)=0$. It means that almost the whole body is considered as a material in which no slips occur. Then, the physical role of slip is transmitted to parts of the body by surface conditions as a kind of the boundary conditions. Such a situation brings about the treatment of description of the martensitic transformation as of the first rank. Slips are viewed to be a more advanced stadium of deformation. Then, for this nanoscale approach, the free energy $F$ has to comprise conditions for which slips are initiated. Consequently, the whole approach to description of martensitic transformation conjugated with slip plasticity is concentrated around the free energy formulation.

The free energy function related to the nanoscale and motivated by properties of $\mathrm{CuAl}$ alloy will be introduced with the help of the following program:

(i) The skeleton of the free energy is based on geometrical objects distinguished in the crystal structure of austenite and martensite. Geometrical objects related to the structure of austenite are:

- Bain axes,

- six different basal planes in undeformed state of austenite,

- twenty four habit planes,

- directions of nonhomogeneous in nanoscale shear which define a set of shear systems,

- a set of slip systems.

Geometrical objects related to the structure of martensite are:

- slip systems,

- paths of martensite-martensite transformation,

- positions of habit and twin type interfaces.

(ii) It is assumed that elastic properties of austenite and martensite are known. The free energy is defined in the neighbourhood of the equilibrium of austenite and martensite as a positive definite quadratic form with appropriate symmetry properties.

(iii) The boundaries of the elastic range for austenite and martensite are given as five-dimensional hypersurfaces in the space of strain $E$. In this region the phase transformation is initiated. Determination of this surface is introduced with the aid of shear systems, slip systems and paths of martensite-martensite transformation.

(vi) The main idea of shaping of the free energy rests on the expression of the graph of the free energy as a fibre bundle spanned on the geometrical skeleton introduced.

(v) The first stage of construction of the free energy rests on defining its part which depends mainly on the strain tensor.

(vi) The second stage consists in introducing relative displacement vectors in order to describe the evolution of shuffles.

(vii) The third stage consists in taking into account internal rotations towards possible habit planes.

(viii) The last stage consists in introducing higher gradients of deformation based on a hierarchy of distinguished directions.

(ix) Dependence of the free energy on temperature and densities of inhomogeneities is introduced by plotting separate elements which define the graph of the free energy with these variables.

We do not realize here whole of this program. We discuss here some aspects of this modelling in order to illustrate the methodology applied. More details related to this program in case of martensitic transformation have been discussed in $[17,18]$.

Let $F_{i j k l}(\mathbf{e})=\frac{\partial^{2} F}{\partial e_{i j} \partial e_{k l}}(\mathbf{e})$. We define a domain of elastic austenite $A$ and the domain of elastic martensite $M_{K}$ by means of the condition that the introduced function is positive definite.

The starting point for the determination of the free energy on the domain $A$ is the positive definite function $\bar{F}_{A}=c_{i j k l} e_{i j} e_{k l}$. This function describes linearly elastic properties of austenite in a neighbourhood $U_{A}$ of $\mathbf{0} \in E, U_{A} \subset$ int $A$. A function of this kind cannot be valid on the whole domain $A$ since the quadratic form $F_{i j k l}$ has to be semipositive definite on $\partial A=\partial S_{A}$. It follows that $\bar{F}_{A}$ should be modified on the domain $A-U_{A}$ in order to change continuously its properties.

Consequently, there exists necessity of defining first $\partial S_{A}$ as an important element of the geometrical skeleton of the free energy constructed. 
Let us introduce the set of hypersurfaces $\mathcal{P}_{I} \subset E, I \in I_{S A}$, defined by the following formula:

$$
\mathcal{P}_{I}=\left\{\mathbf{e}: \frac{\partial}{\partial e_{I 12}}\left(\mathbf{d}_{I 1} \frac{\partial F}{\partial \mathbf{e}} \mathbf{d}_{I 2}\right)=0\right\}
$$

and $\mathcal{P}_{\alpha} \subset E, \alpha \in I_{S L}$, defined by

$$
\mathcal{P}_{\alpha}=\left\{\mathbf{e}: \frac{\partial}{\partial e_{\alpha m n}}\left(\mathbf{m}_{\alpha} \frac{\partial F}{\partial \mathbf{e}} \mathbf{n}_{\alpha}\right)=0\right\} .
$$

We define the set

$$
L_{c}=\left\{\mathbf{e}: \mathbf{e}_{B}=\left\{e_{B i j}\right\}=\left\{p \delta_{i j}\right\}, \mathbf{e}=k \mathbf{e}_{B}, k \in R, p \neq 0\right\} .
$$

We assume that $\mathcal{P}_{I} \cap L_{c}=\emptyset, \mathcal{P}_{\alpha} \cap L_{c}=\emptyset$ for each $I$ and $\alpha$. Formulas (43) and (44) define the set of conditions imposed on the free energy function. In general, we assume that the form of $\mathcal{P}_{I}$ and $\mathcal{P}_{\alpha}$ is known or postulated. Let us also introduce the set $\mathcal{P}_{A}=\bigcup_{I} \mathcal{P}_{I} \cup \bigcup_{\alpha} \mathcal{P}_{\alpha}$.

Let $\mathbf{e}_{D} \in E$ and $\left\|\mathbf{e}_{D}\right\|_{E}=1$ determine a direction in the space $E$. Let us also define the set $\left[\mathbf{e}_{D}\right]=\{\mathbf{e}$ : $\left.\mathbf{e}=a \mathbf{e}_{D}, a \in R_{+}\right\}$. The set of all $\left[\mathbf{e}_{D}\right]$ is denoted by $K_{D}$. With the help of the above defined sets, we introduce functions $\alpha_{a}:\left[\mathbf{e}_{D}\right] \rightarrow R_{+}$defined by $\alpha_{a}(\mathbf{e})=a, \mathbf{e} \in\left[\mathbf{e}_{D}\right], \mathbf{e}=a \mathbf{e}_{D}$ and $a_{A}: K_{D} \rightarrow R_{+}$defined with the help of the formula

$$
a_{A}\left(\left[\mathbf{e}_{D}\right]\right)=\inf _{a}\left\{\alpha_{a}\left(\left[\mathbf{e}_{D}\right] \cap \mathcal{P}_{A}\right)\right\}
$$

By means of the last function, we define $\partial S_{A}$ as

$$
\partial S_{A}=\left\{\mathbf{e}: \mathbf{e}=a \mathbf{e}_{D}, a=a_{A}\left(\left[\mathbf{e}_{D}\right]\right),\left\|\mathbf{e}_{D}\right\|=1\right\} .
$$

Consequently, the remaining sets distinguished in the framework of $\partial S_{A}$ are defined as follows:

$$
\partial S_{I}=\partial S_{A} \cap \mathcal{P}_{I}, \quad \partial S_{\alpha}=\partial S_{A} \cap \mathcal{P}_{\alpha} .
$$

At this moment, we introduce the construction of the graph of the free energy by means of fibre bundle approach [20]. Basis and fibres are defined with the help of the here introduced geometrical skeleton.

First, we will express the domain $A$ as a fibre bundle. Let $A_{I}: E \rightarrow E_{I}$ be a mapping which transforms the strain tensor $\mathbf{e}$ in a given basis $\mathbf{b}$ into the strain tensor $\mathbf{e}_{I}$ expressed in the basis $\mathbf{d}_{I}=\left\{\mathbf{d}_{I \alpha}, \mathbf{d}_{I 1} \times \mathbf{d}_{I 2}\right\}$ and $A_{\alpha}: E \rightarrow E_{\alpha}$ transform the strain tensors into $\mathbf{e}_{\alpha}$ expressed in the basis $\left\{\mathbf{m}_{\alpha}, \mathbf{n}_{\alpha}, \mathbf{m}_{\alpha} \times \mathbf{n}_{\alpha}\right\}$.

Let us introduce sets $N_{I A}=\left\{\mathbf{e}: 0 \leq e_{I 12} \leq e_{I 12}^{*}, e_{I k l}=0\right\}, N_{I S}=\left\{\mathbf{e}: e_{I 12}^{*} \leq e_{I 12} \leq e_{I 12}^{* *}, e_{I k l}=\right.$ $0\}, N_{I M}=\left\{\mathbf{e}: e_{I 12}^{* *} \leq e_{I 12} \leq e_{K 12}, e_{I k l}=0\right\}, N_{I}=N_{I A} \cup N_{I S} \cup N_{I M}$ and additionally

$$
N_{\alpha}=\left\{\mathbf{e}: 0 \leq e_{\alpha m n} \leq e_{\alpha m n}^{*}, e_{\alpha i j}=0\right\} .
$$

Let $N_{o}=N_{I A}-\{\mathbf{0}\}$ and let $h_{A I}\left(e_{I 12}\right): \partial S_{A} \rightarrow f\left(e_{I 12}\right), e_{I 12} \in N_{o}$ be a family of homeomorphisms and the set $\bigcup_{e_{I 12} \in N_{o}} f\left(e_{I 12}\right) \cup\{\mathbf{0}\}=A$ be a decomposition of the domain $A, f\left(e_{I 12}^{\prime}\right) \cap f\left(e_{I 12}^{\prime \prime}\right)=\emptyset$ for $e_{I 12}^{\prime} \neq e_{I 12}^{\prime \prime} \in N_{o}$. Then, $N_{o}=N_{I A}-\{\mathbf{0}\}$ can be viewed as a basis of a fibre bundle, and $f\left(e_{I 12}\right)$ are viewed to be fibres. Finally, the domain $A=\bigcup_{e_{I 12} \in N_{o}} f\left(e_{I 12}\right) \cup\{\mathbf{0}\}=N_{o} \times \partial S_{A} \cup\{\mathbf{0}\}$ is expressed as the fibre bundle or generalized Cartesian product of $N_{o}$ and $\partial S_{A}$.

The graph of the function $F$ is considered in $E \times R$ space. Let us introduce the following sets in this space:

$$
\begin{aligned}
& \bar{B}_{I A}=\left\{\bar{b}_{I A}: \bar{b}_{I A}=\left\{\mathbf{e}, \bar{F}_{A}(\mathbf{e})\right\}, \mathbf{e} \in N_{I A}\right\}, \\
& \bar{B}_{\alpha A}=\left\{\bar{b}_{\alpha A}: \bar{b}_{\alpha A}=\left\{\mathbf{e}, \bar{F}_{A}(\mathbf{e})\right\}, \mathbf{e} \in N_{\alpha A}\right\}
\end{aligned}
$$

and

$$
\begin{aligned}
\bar{F}_{\partial S_{A}} & =\left\{\left\{\mathbf{e}, \bar{F}_{A}(\mathbf{e})\right\}: \mathbf{e} \in \partial S_{A}=f\left(e_{I 12}^{*}\right)\right\}, \\
\bar{F}_{f\left(e_{I 12}\right)} & =\left\{\left\{\mathbf{e}, \bar{F}_{A}(\mathbf{e})\right\}: \mathbf{e} \in f\left(e_{I 12}\right)\right\} .
\end{aligned}
$$

At this stage of considerations, we express the graph of $\bar{F}$ function as a fibre bundle. Thus, we have

$$
G r \bar{F}_{A}=\bigcup_{e_{I 12} \in N_{o}} \bar{F}_{f\left(e_{I 12}\right)}=\bar{B}_{I A} \times \bar{F}_{\partial S_{A}}
$$


with projection in the bundle $\pi\left(\bar{F}_{f\left(e_{I 12}\right)}\right)=\left\{\mathbf{e}, \bar{F}_{A}(\mathbf{e})\right\} \in \bar{B}_{I A},\left(A_{I}(\mathbf{e})\right)_{12}=e_{I 12} . \bar{F}_{A}$ can be expressed alternatively by $\operatorname{Gr} \bar{F}_{A}=\bar{B}_{\alpha A} \times \bar{F}_{\partial S_{A}}$.

We postulate that $\operatorname{Gr} \bar{F}_{A}$ defined with the help of (50) contains also all $\bar{B}_{I A}, \bar{B}_{\alpha A}$ and $\bar{F}_{\partial S}, \bar{F}_{f\left(e_{I 12}\right)}$.

The function $\bar{F}_{A}$ expresses elastic properties of austenite for $\mathbf{e} \in U_{A}$ and has to be modified for $\mathbf{e} \in A-U_{A}$ in order to gradually attain appropriate properties on $\partial S_{A}$. The concept of this modification consists in adding a correction function $C$ determined on $A-U_{A}$. Accordingly, the modified function $F_{A}$ takes the form

$$
F_{A}=\left\{\begin{array}{ll}
\bar{F}_{A}, & \mathbf{e} \in U_{A} \\
\bar{F}_{A}+C, & \mathbf{e} \in A-U_{A}
\end{array} .\right.
$$

The graph of the function $C$ is defined as follows:

$$
G r C=\left\{\begin{array}{ll}
\{\mathbf{e}, 0\}, & \mathbf{e} \in U_{A}, e_{I 12} \leq \bar{e}_{I 12}=N_{I A} \cap \partial U_{A} \\
\left\{\mathbf{e}, C_{e_{I 12}}(\mathbf{e})\right\}, & \mathbf{e} \in f\left(e_{I 12}\right), \bar{e}_{I 12}<e_{I 12}<e_{I 12}^{*}
\end{array} .\right.
$$

We assume that the following conditions are fulfilled by the modified function:

$$
\begin{aligned}
& \frac{\partial^{2}\left(\bar{F}_{A}+C\right)}{\partial \mathbf{n}^{2}\left(e_{I 12}\right)}(\mathbf{e})=0, \quad \mathbf{e} \in \partial S_{A} \cap N_{I A}, \\
& \frac{\partial^{2}\left(\bar{F}_{A}+C\right)}{\partial \mathbf{n}^{2}\left(e_{\alpha m n}\right)}(\mathbf{e})=0, \quad \mathbf{e} \in \partial S_{A} \cap N_{\alpha}
\end{aligned}
$$

where $\frac{\partial^{2}}{\partial \mathbf{n}^{2}\left(e_{I 12}\right)}, \frac{\partial^{2}}{\partial \mathbf{n}^{2}\left(e_{\alpha m n}\right)}$ represent differentiation in tangent to $N_{I A}$ and $N_{\alpha}$ directions in the given point $\mathbf{e} \in \partial S_{A} \cap N_{I A}$ or $\mathbf{e} \in \partial S_{A} \cap N_{\alpha}$, respectively. Furthermore, $F_{A i j k l}$ is semipositive definite on the whole $\partial S_{A}$.

Let $B_{I A}=\left\{b_{I A}: b_{I A}=\left\{\mathbf{e}, F_{A}(\mathbf{e})\right\}: \quad \mathbf{e} \in N_{I A}\right\}, F_{f\left(e_{I 12}\right)}=\left\{\left\{\mathbf{e},\left(\bar{F}_{A}+C\right)(\mathbf{e})\right\}: \quad \mathbf{e} \in f\left(e_{I 12}\right)\right\}$ and $F_{\partial S_{A}}=\left\{\left\{\mathbf{e},\left(\bar{F}_{A}+C\right)(\mathbf{e})\right\}: \quad \mathbf{e} \in \partial S_{A}\right\}$. Then, the graph of the function $F_{A}$ can be expressed as $G r F_{A}=B_{I A} \times F_{\partial S_{A}}=\bigcup_{\mathbf{e} \in N_{0}} F_{f\left(e_{I 12}\right)}$.

As similar construction will be carried out for the free energy function determined on the domain $M_{K}$. Situation becomes more complicated since criteria of the determination of $\partial S_{K}$ are not so clear. We have assumed that $\partial S_{K}$ is determined by its general form given by (37).

We define the set $L_{K c}$ accompanied by the isotropic part of strain of $K$ th martensite variant as follows:

$$
L_{K c}=\left\{\mathbf{e}: \tilde{e}_{B K i j}=p \delta_{i j}, \tilde{\mathbf{e}}_{K}=k \tilde{\mathbf{e}}_{B K}, p \neq 0, k \in R\right\}
$$

where $p$ is a given value.

Determination of $\mathcal{P}_{K \beta}$ is related to slip systems introduced for the $K$ th variant of martensite $\left\{\mathbf{m}_{K \beta}, \mathbf{n}_{K \beta}\right\}$ and the strain $\tilde{\mathbf{e}}_{K}$ defined by (39). Consequently, we introduce the following definition:

$$
\mathcal{P}_{K \beta}=\left\{\mathbf{e}: \frac{\partial}{\partial e_{K \beta m n}}\left(\mathbf{m}_{K \beta} \frac{\partial F}{\partial \tilde{\mathbf{e}}_{K}} \mathbf{n}_{K \beta}\right)=0\right\} .
$$

The hypersurface $\mathcal{P}_{K A}$ related to the path connected with $K$ th martensite variant to austenite transformation for $I \subset K$, is defined with the help of shear systems introduced for austenite and transformed to martensite as

$$
\mathcal{P}_{K A}=\left\{\mathbf{e}: \frac{\partial}{\partial e_{I 12}}\left(\mathbf{d}_{I 1}\left(\mathbf{R}_{K}\right) \frac{\partial F}{\partial \mathbf{e}} \mathbf{d}_{I 2}\left(\mathbf{R}_{K}\right)\right)=0\right\} .
$$

This hypersurface is defined in the same way as (43). $\mathcal{P}_{K A}$ contains $A_{I}^{-1}\left(\mathbf{e}_{I}^{* *}\right)$ while $\mathcal{P}_{I}$ contains $A_{I}^{-1}\left(\mathbf{e}_{I}^{*}\right)$.

As it was discussed previously, there is also a possible transformation of $K$ th to $K^{\prime}$ th martensite variant. However, this does not happen in one slip system. Therefore, we define first a one-dimensional path $G\left(K, K^{\prime}\right) \subset E$ which joins equilibrium states of martensities determined by $\mathbf{e}_{K}$ and $\mathbf{e}_{K^{\prime}}$. We try to define $\mathcal{P}_{K K^{\prime}}$ with the help of $G\left(K, K^{\prime}\right)$. We introduce some simplified assumptions. First, $\mathcal{P}_{K K^{\prime}}$ is flat, and it is a five-dimensional hyperplane.

In the considered case, $\mathcal{P}_{K K^{\prime}}$ is expressed in a general form as follows:

$$
\mathcal{P}_{K K^{\prime}}=\left\{\mathbf{e}: D_{i j} e_{i j}+D_{o}=0\right\} .
$$


We have to determine six coefficients $D_{i j}$. There are some premises in order to do it. First, $\mathcal{P}_{K K^{\prime}} \| L_{K c}$ gives one equation related to $D_{i j}$. It is expected that a value $\mathbf{e}^{*} \in G\left(K, K^{\prime}\right) \cap \partial S_{K}$ is attainable by experiments for a well-defined path $G\left(K, K^{\prime}\right)$. Other constants could be a result of analysis of transversality conditions between $G\left(K, K^{\prime}\right)$ and $\mathcal{P}_{K K^{\prime}}$ in the point $\mathbf{e}^{*}$.

Let us define the set $\mathcal{P}_{K}=\bigcup_{\beta} \mathcal{P}_{K \beta} \cup \mathcal{P}_{K A} \cup \bigcup_{K^{\prime}} \mathcal{P}_{K K^{\prime}}$ by analogy to considerations related to austenite.

In order to define $\partial S_{K}$, we introduce the function

$$
a_{K}\left(\left[\tilde{\mathbf{e}}_{K D}\right]\right)=\inf _{a}\left\{\alpha_{a}\left(\left[\tilde{\mathbf{e}}_{K D}\right] \cap \mathcal{P}_{K}\right)\right\} .
$$

Thereby, we have an analogy of $\mathcal{P}_{K}$ with $P_{A}$ for austenite. Consequently, $\left\{\mathbf{m}_{K \beta}, \mathbf{n}_{K \beta}\right\}, \beta \in I_{S L K}$ have, by assumption, the status of well-defined slip systems in accordance with the form of the free energy.

Finally, we define

$$
\partial S_{K}=\left\{\mathbf{e}: \tilde{\mathbf{e}}_{K}=a \tilde{\mathbf{e}}_{K D}, a=a_{K}\left(\left[\tilde{\mathbf{e}}_{K D}\right]\right),\left\|\tilde{\mathbf{e}}_{K D}\right\|=1\right\}
$$

and next

$$
\partial S_{K \beta}=\partial S_{K} \cap \mathcal{P}_{K \beta}, \quad \partial S_{K A}=\partial S_{K} \cap \mathcal{P}_{K A}, \quad \partial S_{K K^{\prime}}=\partial S_{K} \cap \mathcal{P}_{K K^{\prime}}
$$

At this moment, we are able to introduce the procedure of defining the graph of the free energy on $M_{K}$ domain by means of fibre bundles. Let $N_{\beta K}=\left\{\mathbf{e}: 0 \leq e_{K \beta m n} \leq e_{K \beta m n}^{*}\right\}$ and $N_{\beta K}-\left\{\mathbf{0}_{K}\right\}=$ $N_{K o}$. Let $h_{K}\left(e_{K \beta m n}\right): \partial S_{K} \rightarrow f\left(e_{K \beta m n}\right), e_{K \beta m n} \in N_{K o}$ be family of homeomorphisms. Then, $M_{K}=$ $\bigcup_{e_{K \beta m n} \in N_{K o}} f\left(e_{K \beta m n}\right) \cup\left\{\mathbf{0}_{K}\right\}=N_{K o} \times \partial S_{K} \cup\left\{\mathbf{0}_{K}\right\}$ is expressed as a generalization of the Cartesian product of $N_{K o}$ and $\partial S_{K}$.

We introduce the function $\bar{F}_{K}=c_{K i j k l} \tilde{e}_{K i j} \tilde{e}_{K k l}$ which describes the elastic properties of the $K$ th variant of martensite in $U_{K}$ domain, $\mathbf{0}_{K} \in U_{K} \subset$ int $M_{K}$,

$$
\begin{aligned}
\bar{B}_{\beta K} & =\left\{\bar{b}_{K}: \bar{b}_{K}=\left\{\mathbf{e}, \bar{F}_{K}(\mathbf{e})\right\}, \mathbf{e} \in N_{\beta K}\right\}, \\
\bar{F}_{\partial S_{K}} & =\left\{\left\{\mathbf{e}, \bar{F}_{K}(\mathbf{e})\right\}: \mathbf{e} \in \partial S_{K}\right\}, \\
\bar{F}_{f\left(e_{K \beta m n}\right)} & =\left\{\left\{\mathbf{e}, \bar{F}_{K}(\mathbf{e})\right\}: \mathbf{e} \in f\left(e_{K \beta m n}\right)\right\} .
\end{aligned}
$$

With the help of these sets, we define the graph of $\bar{F}_{K}$ as follows:

$$
G r \bar{F}_{K}=\bigcup_{\mathbf{e} \in N_{K o}} \bar{F}_{f\left(e_{K \beta m n}\right)}=\bar{B}_{\beta K} \times \bar{F}_{\partial S_{K}} .
$$

The next stage of defining $F_{K}$ consists in modification $\bar{F}_{K}$ on the $M_{K}-U_{K}$ domain. This is carried out in a similar way as for austenite by

$$
F_{K}= \begin{cases}\bar{F}_{K}, & \mathbf{e} \in U_{K} \\ \bar{F}_{K}+C_{K}, & \mathbf{e} \in M_{K}-U_{K}\end{cases}
$$

where the graph of the function $C_{K}$ is defined by

$$
G r C_{K}=\left\{\begin{array}{ll}
\{\mathbf{e}, 0\}, & \mathbf{e} \in U_{K} \\
\left\{\mathbf{e}, C_{K}(\mathbf{e})\right\}, & \mathbf{e} \in f\left(e_{K \beta m n}\right), \quad \bar{e}_{K \beta m n} \leq e_{K \beta m n} \leq e_{K \beta m n}^{*} .
\end{array} .\right.
$$

We assume that $F_{K}$ satisfies the following conditions on $\partial S_{K}$ :

$$
\begin{aligned}
& \frac{\partial^{2}\left(\bar{F}_{K}+C_{K}\right)}{\partial \mathbf{n}^{2}\left(e_{K \beta m n}\right)}(\mathbf{e})=0, \quad \mathbf{e} \in \partial S_{K} \cap N_{\beta K}, \\
& \frac{\partial^{2}\left(\bar{F}_{K}+C_{K}\right)}{\partial \mathbf{n}^{2}\left(e_{K 12}\right)}(\mathbf{e})=0, \quad \mathbf{e} \in \partial S_{K} \cap N_{I M}, \\
& \frac{\partial^{2}\left(\bar{F}_{K}+C_{K}\right)}{\partial \mathbf{n}^{2}\left(G\left(K, K^{\prime}\right)\right)}(\mathbf{e})=0, \mathbf{e} \in \partial S_{K} \cap G\left(K, K^{\prime}\right) .
\end{aligned}
$$

Furthermore, the quadratic form $F_{K i j k l}$ is semipositive definite on the whole domain $\partial S_{K}$. 
Let $B_{K}=\left\{b_{K}: b_{K}=\left\{\mathbf{e}, F_{K}(\mathbf{e})\right\}, \mathbf{e} \in N_{\beta K}\right\}, F_{f\left(e_{K \beta m n}\right)}=\left\{\left\{\mathbf{e},\left(\bar{F}_{K}+C_{K}\right)(\mathbf{e})\right\}: \mathbf{e} \in f\left(e_{K \beta m n}\right)\right\}$ and $F_{\partial S_{K}}=\left\{\left\{\mathbf{e},\left(\bar{F}_{K}+C_{K}\right)(\mathbf{e})\right\}: \mathbf{e} \in \partial S_{K}\right\}$. Finally, the graph of the free energy $F_{K}$ on the domain $M_{K}$ is defined by $G r F_{K}=B_{K} \times \partial F_{\partial S_{K}}=\bigcup_{\mathbf{e} \in N_{K o}} F_{f\left(e_{K \beta m n}\right)}$.

The form of the free energy function $F_{S}$ defined on the $S$ domain is more generally determined. It follows from the fact that it is necessary to have more detailed information about properties of this function on the set $S$.

Therefore, we assume only general properties for this part of the free energy. Consequently, we assume that the following conditions are satisfied by this function:

$$
\begin{aligned}
\left.F_{S}\right|_{N_{I S}} & =B_{I S},\left.\quad F_{S}\right|_{\partial S_{A}}=\left.F_{A}\right|_{\partial S_{A}},\left.\quad F_{S}\right|_{\partial S_{K}}=\left.F_{K}\right|_{\partial S_{K}}, \\
\left.\frac{\partial F_{S}}{\partial \mathbf{e}}\right|_{\partial S_{A}} & =\left.\frac{\partial F_{A}}{\partial \mathbf{e}}\right|_{\partial S_{A}},\left.\quad \frac{\partial F_{S}}{\partial \mathbf{e}}\right|_{\partial S_{K}}=\left.\frac{\partial F_{K}}{\partial \mathbf{e}}\right|_{\partial S_{K},} \\
\left.\frac{\partial^{2} F_{S}}{\partial \mathbf{e} \partial \mathbf{e}}\right|_{\partial S_{A}} & =\left.\frac{\partial^{2} F_{A}}{\partial \mathbf{e} \partial \mathbf{e}}\right|_{\partial S_{A}},\left.\quad \frac{\partial^{2} F_{S}}{\partial \mathbf{e} \partial \mathbf{e}}\right|_{\partial S_{K}}=\left.\frac{\partial^{2} F_{K}}{\partial \mathbf{e} \partial \mathbf{e}}\right|_{\partial S_{K}}
\end{aligned}
$$

where $B_{I S}$ should be postulated.

Finally, the part of the free energy $F_{E}$ which depends mainly on the strain tensor $\mathbf{e}$ has the form

$$
F_{E}= \begin{cases}F_{A}, & \mathbf{e} \in A \\ F_{K}\left(\mathbf{R}_{K}\right), & \mathbf{e} \in M_{K} \\ F_{S}, & \mathbf{e} \in S\end{cases}
$$

The formula (69) determines the form of the free energy for which austenite and martensite coexist in equilibrium states. However, if temperature increases, we obtain a pseudoelasticity phenomenon. It lies in the necessity of maintaining stress in order to have stable martensite. It means that no martensite exists if stress is equal to zero. Further increasing of temperature leads to elastic austenite only. On the other hand, decreasing of temperature brings about vanishing austenite state. Then, elastic martensite variants exist only. Dependence of the free energy on temperature should reflect above mentioned changes.

In general, the dependence of $F$ on temperature is introduced by $c_{i j k l}(T), c_{K j i k l}(T), C(T), C_{K}(T), U_{A}(T)$, $U_{K}(T)$ and $\partial S(T)$.

These quantities can also be plotted with other kinds of variables. We can introduce for instance inhomogeneities described by $\rho$ which can affect additionally conditions for slip initiation.

Relative displacement vectors describing shuffles and internal rotations of martensite variants described by variables (34), (35) need additional terms of the free energy $[17,18]$. We do not discuss here it for simplicity. However, they are important. Relative displacement vectors determine a kind of internal rotation which the previously formed structure determined by the strain tensor can attain. In this case, we have to do with bifurcation on the path of relative displacement vectors evolution and also on the path of internal rotation evolution $[17,18]$. Furthermore, the relative displacement vectors can introduce new quality. They are able to describe two-path stress-induced martensitic transformation [21].

Nonlocal effects can additionally be introduced. Such a description depends on higher order derivatives of the introduced variables. In nanoscale such an approach can be justified. This is the case especially when martensitic interfaces are moving.

Summarizing we have discussed a rather complex method of shaping of the free energy function graph. However, this method is clear physically since geometrical elements which define the fibre bundle have clear physical interpretation which seems to be an advantage in comparison with the Landau method. Furthermore, the number of phenomena which can be modelled on this way considerably increases. Thereby, we have attained both previously mentioned aims: clear interpretation of geometry of the free energy graph and unificational properties of this function.

Let us also note that the fibre manifold as graph of the function can be generated by numerical simulations. Thereby, this method seems to be convenient for the creation of conditions for numerical simulations of the whole material behaviour.

Considerable number of constants of this model can be difficult for identification on experimental way. However, when we apply molecular dynamics equations as EDS, then constants could be calculated by numerical simulations. Furthermore, considerable amount of data provided by molecular dynamics simulations could be collected in form of particular nanoscale models with identified on numerical way constants. 


\subsection{Role of the free energy for unified description of various phenomena}

When processes in the material are associated with small deformation, then usually we have to do with elasticity. However, when deformation increases various phenomena can appear. Consequently, many phenomena can be initiated on the boundary of the elastic region. Thereby, defining the set $\partial S_{A}$ is of key importance for the free energy related to the considered set of phenomena.

We have defined previously hypersurfaces $\mathcal{P}_{I}$ and $\mathcal{P}_{\alpha}$ as in (43) and (44). They define parts of the set $\partial S_{A}$. By this step, we have unification of martensitic transformation with slip plasticity.

We can introduce by analogy fracture systems, for instance, defined by vectors characterizing tension within the material. Then, the corresponding hypersurface $\mathcal{P}_{F r}$ could also be a part of the set $\partial S_{A}$ [22]. Then, on this region, brittle fracture could be initiated.

Summarizing, by considerations of the free energy graph as a manifold, we can specify geometrical elements responsible for the description of various phenomena. Therefore, this method of defining the free energy function seems to be convenient also for unification of various descriptions related to the same nanoscale level.

\section{On the possibility of extension of the multiscale modelling towards scale lower than atomistic one}

We understand transition to atomistic scale within mechanics of materials usually by application of molecular dynamics method. However, this method is based on the averaged effect of electronic processes leading to the determination of potential describing interactions between nuclei. In this case, electronic processes are considered in fact as relatively less important than motion of nuclei.

Let us notice that long-lasting biological evolution leads to homeothermal systems. This means that nuclei motions are in fact subordinated to the remaining part of the dynamical system. This remaining part corresponds to electronic structure. It means that physical description of electronic processes is perhaps not sufficiently good within existing theories. Let us mention also that the existence of biological evolution as such is not described by fundamental physical theories.

The last statements suggest that also mechanics of materials perhaps depends to a larger degree on an appropriate description of electronic processes as well as on models of conjugation of electronic processes and nuclei motion. In particular, molecular dynamics method should then be modified in order to take into account particular electronic effects.

In order to overcome the mentioned difficulties of fundamental theories, vacuum medium mechanics is introduced by the author. Effects of the vacuum medium mechanics on the description of biological processes including driven force for biological evolution based on elementary particle processes are discussed in [23-25] (see also references in these papers related directly to vacuum medium mechanics).

Let us notice that currently developed biomechanics applies descriptions corresponding to various biological structures. These descriptions were usually developed previously for mechanics of materials. Let us mention mechanics of composites for instance. Thereby, the described biological structures are not seen within description as living structures. This means that physics of corresponding objects is considered in a too simplified way.

Let us notice also that biological evolution with the driven force placed on the most fundamental level is able to organize structures which in turn are able to response on large-scale external interactions including usual loading as in engineering constructions. Then, biological structures are elaborated by evolution towards resistance against a large variety of external loadings related also to various scales. It seems that biomechanics should be able to describe just such effects having also in its description the awareness that describing structures are living ones. This in turn means that we should have at our disposal a multiscale method of modelling which takes into account also the most fundamental interactions.

Within this Sect. 1 we state that multiscale method of modelling presented in this work is formulated on a way which is able to take into account in future also results of vacuum medium mechanics by formal incorporation of it into one theoretical scheme. This statement seems perhaps too speculative. However, considerable efforts already have been done in order to make such a possibility real in future [23-25].

Consequently, by steps presented in this paper, we try to provide the context for construction in future of unified mechanics of condensed matter in cooperation with various type materials. This leads also to a multidisciplinary character of this kind of unified mechanics. It seems that nanotechnology needs just this kind of theory. 


\section{Summarizing comments on the methodology applied to the formulation of unified mechanics of materials}

Let us describe concisely the procedure of constructing a multiscale model of mechanics of materials accentuating methodology related to various steps.

Let us assume that we have at our disposal the possibility of molecular dynamics simulations of behavior of a material at the atomic scale. It means that we have determined an elementary dynamical system (EDS) which means in turn that we have determined equations describing the evolution of atomic nuclei in a determined electronic potential.

We would like to obtain a nanoscale model of this material. This means that we should determine corresponding skeletal dynamical system SDS. This needs in turn determination of collection of dynamical systems within EDS. The option of dynamical subsystems should be consistent with the scale of SDS. We assume that the nanoscale model should be a continuum model. Then, we should determine a set of geometrical objects $\left\{K_{h}\right\}$ which next approximate our body. The geometrical objects $\left\{K_{h}\right\}$ should be consistent with distinguished collection of dynamical systems in order to obtain in a possible natural way a mapping $G_{K}$ leading to an appropriate approximation of our body.

Let us notice that we try to construct a continuum model with finite-dimensional fields. This means that we do not need the discretization procedure usually applied to continuum mechanics models. Direct finitedimensional modelling is associated with the methodology of approximation of physical processes.

This kind of approximation differs from continuum models with infinite-dimensional fields. Let us note that frequently continuum models have not determined the scale of averaging. However, they have a precisely developed mathematical formulation. Let us mention models where micropolar effects are taken into account. We have then a precise mathematical apparatus, and we are able to obtain theorems within such theories. However, then connection with reality is not well maintained. It is difficult for instance to interpret rotations considered in fact in infinite small scale. Then, various proofs within formalism also are not bounded by minimal size related to scale. Such proofs are formally correct, but their connection with reality is difficult to maintain.

It seems that our cognition of reality is efficient only for finite-dimensional description. This is so since our neural network is finite dimensional. Therefore, finite-dimensional approximation of real processes seems to be more appropriate. This is so also since our continuum model is simpler than finite-dimensional molecular dynamics equations. Therefore, corresponding fields of SDS should have smaller dimension than corresponding EDS. Infinite-dimensional descriptions of reality are perhaps a challenge for our brain related to a future.

Consequently, within our methodology, we postulate approximation of real processes in a continuum within sets $K_{h}$ by appropriate option of variables in a consistent way with previously assumed EDS or postulated directly in case when the nanoscale model will be considered as EDS. Then, variables describe a state within $K_{h}$ and continuum fields approximate these states in each point of the continuum. By such a step, we can consider various interactions including also nonlocal effects for instance.

The option of variables for a nanoscale model depends on the phenomenon which we would like to describe. This can be done by direct postulate in case when our nanoscale model is considered as EDS. In case when our model is considered as SDS, variables can be postulated with the aid of motivations given by mappings $\pi$ which provide connections with EDS.

Constitutive equations are also associated with quantities introduced on $K_{h}$. In particular, they can express nonlocal interactions. This in turn depends on the introduction of higher gradients. Higher gradients can be introduced in formal way by means of mappings $a_{h}$ which are next useful for defining continuum fields.

Sometimes more fine effects related even to smaller scale should be taken into account. We can apply then internal state variables which will create in part the SDS.

Let us note that sometimes we are forced to describe phenomena which are not homogeneously distributed within $K_{h}$. Let us mention slip surface for instance. Then, $K_{h}$ can be divided into parts after exceeding some critical conditions by processes. Then, we have to realize the transition to a qualitatively new model. Then, some critical conditions are responsible for correct cooperation of various nanoscale models.

The critical conditions can be frequently expressed by means of the free energy. Therefore, we have discussed previously the methodology of constructing this function taking into account its unificational role for various phenomena on the same level of description related to a given scale. Consequently, the previously formulated method of determining the free energy should be adjusted to collection of dynamical system by appropriate redefinition of variables taking into account the discrete character of description, introduction of gradients by means of mappings $a_{h}$ and creating appropriate relations with continuum fields introduced. 
Let us also notice that from the finite size of $K_{h}$ follows that application of additional variables for the description of the state of material within $K_{h}$ seems to be more convenient than application of higher gradients.

All above steps should lead to determination of SDS for nanoscale models. Then, corresponding SDS together with mappings $\pi$ should take place in elaboration of the reduced dynamical system RDS by means of EDS. It is admissible to consider the postulated nanoscale model as independent with constants determined by some experiments. Such a model can be also considered as EDS for more averaged models of inelastic deformation.

One suggests that more averaged models, frequently applied in engineering, should be three-scale models. First level is atomic one. The second level of description would be represented by nanoscale models. Then, more averaged models would be elaborated with the aid of nanoscale EDS. This means that the dimension of fields for engineering models of mechanics of materials should be considerably lower than that one related to nanoscale. As a result of this, no discretization procedure would be applied. The larger-scale model would obtain physical interpretation following from lower scale. All critical conditions for transition between models of the same scale would be obtained also with the aid of premises better justified physically and following from smaller scale. All this should provide a context for the determination of the range of validity of various models for the same scale and possibility of numerical cooperation between them.

\section{Conclusions}

In this paper, we have discussed a way of development of the rational mechanics which would take into account the whole material starting from atomistic scale by transition sequentially to larger and larger scale. By this approach, we have a hope to elaborate a unified mechanics of materials which would provide a consistent theoretical framework for future numerical simulations of the whole material. In other words, we tend to a theory with large range of validity.

This property should be attained by the possibility of transition between various scales as well as by the determination of range of the validity of submodels by a set of critical conditions. Then, transition between various models within the same scale could be also possible. Such conditions ensure that we need not to stop numerical simulations in case of violating the critical condition of validity for a given model.

This approach is aimed at introducing of engineering for various scales including nanoscale in a systematical way. The term "systematical way" means that we would be able to realize a design of devices, constructions and manufacturing processes by means of a unified theory and complex numerical simulations. Then, design criteria also could be defined within the theoretical approach in relation to various scales.

Such an engineering is observed in biological systems where molecular structures are undoubtedly designed in an unknown manner using criteria following from various scales. We should tend towards obtaining such possibilities.

Open Access This article is distributed under the terms of the Creative Commons Attribution License which permits any use, distribution, and reproduction in any medium, provided the original author(s) and the source are credited.

\section{References}

1. Truesdell, C.: A First Course in Rational Mechanics. Academic Press, Baltimore (1972)

2. Penrose, O.: Foundations of statistical mechanics. Rep. Prog. Phys. 42, 1937 (1979)

3. Balescu, R.: Equilibrium and Nonequilibrium Statistical Mechanics. Wiley, London (1975)

4. Mura, T.: Micromechanics of Defects in Solids. Martinus Nijhoff Publishers, Boston (1982)

5. Nemat-Nasser, S., Hori, M.: Micromechanics: Overall Properties of Heterogeneous Materials. Elsevier, Amsterdam (1999)

6. Perzyna, P.: Thermodynamics of Inelastic Materials. PWN, Warszawa (1978)

7. Hirsch, M.W., Smale, S.: Differential Equations, Dynamical Systems and Linear Algebra. Academic Press, New York (1974)

8. Haken, H.: Synergetics. Springer, Berlin (1977)

9. Kaczmarek, J.: Multiscale modelling in mechanics of materials, 514/1473/2000. Reports of IMP PAN Gdańsk, $514 / 1473 / 2000$

10. Kaczmarek, J.: Collection of dynamical systems with dimensional reduction as a multiscale method of modelling for mechanics of materials. Interact. Multiscale Mech. 3, 1-22 (2010)

11. Kaczmarek, J.: A method of integration of molecular dynamics and continuum mechanics for solids. TASK Q. 6, 253-271 (2002)

12. Kaczmarek, J.: A stochastic continuum dynamical system based on a dimensional reduction procedure. Bull. Pol. Acad. Sci. 46, 149-162 (1998) 
13. Barsch, G.R., Krumhansl, J.A.: Twin boundaries in ferroelastic media whithout interface dislocations. Phys. Rev. Lett. 53, 1069-1072 (1984)

14. Barsch, G.R., Krumhans, J.A.: Nonlinear and nonlocal continuum model of transformation precursors in martensities. Met. Trans. 19A, 761-775 (1988)

15. Liakos, J.K., Saunders, G.A.: Application of the Landau theory to elastic phase transitions. Phil. Mag. A 46, 217-242 (1982)

16. Cohen, M., Olson, G.B., Clapp, P.C.: On the classification of displacive transformations. In: Proceedings of International Conference on ICOMAT (1979)

17. Kaczmarek, J.: A model of the free energy for materials which undergo martensitic phase transformations with shuffles. Int. J. Eng. Sci. 32, 369-384 (1994)

18. Kaczmarek, J.: A thermodynamical description of the martensitic transformation. A model with small volume of averaging. Arch. Mech. 50, 53-81 (1998)

19. Kaczmarek, J.: A nanoscale model of crystal plasticity. Int. J. Plast. 19, 1585-1603 (2003)

20. von Westenholtz, C.: Differential Forms in Mathematical Physics. North Holland, Amsterdam (1981)

21. Kaczmarek, J.: Stress-strain relation for materials which undergo two-path stress-induced displacive phase transition. Int. J. Eng. Sci. 29, 883-888 (1991)

22. Kaczmarek, J., Ostachowicz, W.: A description of damage based on nanoscale modelling of fracture. Key Eng. Mat. 293-294, 235-244 (2005)

23. Kaczmarek, J.: On a structure of mathematical theory which reflects multiscale aspects and integrity of biological systems I. Far East J. Appl. Math. 76, 79-117 (2013)

24. Kaczmarek, J.: On a structure of mathematical theory which reflects multiscale aspects and integrity of biological systems II. Far East J. Appl. Math. 77, 77-111 (2013)

25. Kaczmarek, J.: On emergence of intelligence in biological systems and methods of modelling of reality necessary for development of theoretical biology. Far East J. Appl. Math. 75, 101-150 (2013) 